\title{
Anther dehiscence is regulated by gibberellic acid in yellow lupine (Lupinus luteus L.)
}

\author{
Katarzyna Marciniak* (iD and Krzysztof Przedniczek
}

\begin{abstract}
Background: Anther dehiscence resulting in the release of pollen grains is tightly regulated in a spatiotemporal manner by various factors. In yellow lupine (Lupinus luteus L.), a species that shows cleistogamy, the anthers split before the flowers open, but the course and regulation of this process are unknown. The specific control of anther development takes place via hormonal pathways, the wide action of which ensures reproductive success. In our previous research concerning flower and early pod development in yellow lupine, we showed that the lowest transcript level of LIDELLA1, a main repressor of gibberellin (GA) signalling, occurs approximately at the time of anther opening; therefore, the main purpose of this study was to precisely investigate the gibberellic acid $\left(\mathrm{GA}_{3}\right)^{-}$ dependent regulation of the anther dehiscence in this species.

Results: In this paper, we showed the specific changes in the yellow lupine anther structure during dehiscence, including secondary thickening in the endothecium by lignocellulosic deposition, enzymatic cell wall breakdown at the septum/stomium and cell degeneration via programmed cell death (PCD), and identified several genes widely associated with this process. The expression profile of genes varied over time, with the most intense mRNA accumulation in the phases prior to or at the time of anther opening. The transcriptional activity also revealed that these genes are highly coexpressed and regulated in a GA-dependent manner. The cellular and tissue localization of $\mathrm{GA}_{3}$ showed that these molecules are present before anther opening, mainly in septum cells, near the vascular bundle and in the endothecium, and that they are subsequently undetectable. $\mathrm{GA}_{3}$ localization strongly correlates with the transcriptional activity of genes related to GA biosynthesis and deactivation. The results also suggest that $\mathrm{GA}_{3}$ controls LIGAMYB expression via an LIMIR159-dependent pathway.

Conclusions: The presented results show a clear contribution of $\mathrm{GA}_{3}$ in the control of the extensive anther dehiscence process in yellow lupine. Understanding the processes underlying pollen release at the hormonal and molecular levels is a significant aspect of controlling fertility in this economically important legume crop species and is of increasing interest to breeders.
\end{abstract}

Keywords: Anther dehiscence, Gibberellins, Endothecium, Secondary thickening, Septum/stomium rupture, Degeneration via PCD, Yellow lupine, Legumes

\footnotetext{
* Correspondence: marciniak@umk.pl

Faculty of Biological and Veterinary Sciences, Department of Plant Physiology and Biotechnology, Nicolaus Copernicus University, Lwowska 1 St, 87-100 Toruń, Poland
} 


\section{Background}

The critical factors in plant reproduction are the proper development of stamens and the release of viable pollen grains, followed by pollination, fertilization and fruit/pod setting and development. This is particularly important in crop plants, including legumes, due to the impact on yield. Knowledge of stamen development is increasing, but most of the information comes from analyses of male-sterile mutants in Arabidopsis thaliana and rice (Oryza sativa) [1-5]. Fourteen early and late phases of stamen development have been identified in the model plant $A$. thaliana $[1,6,7]$. The fundamental and extensive processes that occur in maturing stamens is anther dehiscence, which generally consists of the following stages: (I) expansion of the endothecium and deposition of materials responsible for wall thickening in endothecial and connective cells; (II) degeneration of the tapetum and middle layer (stages 7-11); (III) enzymatic opening of the septum between two locules and its progressive degeneration (stages 11-12); (IV) breakage of the stomium formed from modified epidermal cells (stage 12); and (V) the release of pollen grains from the locules during stage 13. Finally, the anthers senesce and are separated from flowers (stages 14a-14c) [1, 2]. Many species, such as $A$. thaliana, tobacco (Nicotiana tabacum) and rice, exhibit comparable courses of anther dehiscence $[1,8-10]$. The differentiation and degeneration of the septum (circular cell cluster in $N$. tabacum) and stomium are conserved [8]; however, developmental differences exist, e.g., in the anther structure or type of endothecial thickening $[11,12]$. Much evidence has demonstrated the crucial role of secondary cell wall thickening in pollen release. It has been suggested that this is closely related to the delivery of force to disrupt stomium cells $[2,4,11]$. The key components of secondary cell walls are lignin, cellulose and hemicelluloses (xylan and glucomannan). Lignin consists of three subunits, namely, p-hydroxyphenyl $(\mathrm{H})$, guaiacyl $(\mathrm{G})$ and syringyl (S), and the most important enzyme catalysing the last step of lignin biosynthesis is cinnamyl alcohol dehydrogenase (CAD) [13]. In $A$. thaliana, two $C A D$ genes $(C A D C, C A D D)$ have been identified, and mutants of these genes show the absence of lignin in the endothecium, leading to anther indehiscence [14]. Among the genes involved in secondary cell wall formation, IRRE GULAR XYLEM1/6/8 (IRX1/6/8) can also be distinguished [15-17]. IRX1, with the alternative name cellulose synthase A catalytic subunit 8 (CesA8), is required for the synthesis of cellulose [16]; IRX6 (synonym COBRA-like4, COBL4) encodes a member of the COBRA family that is needed for the deposition of cellulose whereas IRX8 (synonym galacturonosyltransferase 12, GAUT12) belongs to the CAZy family of glycosyl transferase (GT8), which is involved in the deposition of xylan and lignin in secondary cell walls $[15,18]$. One of the major events in anther dehiscence is pectin degradation leading to cell separation, which involves enzymes such as polygalacturonases (PGs). QUARTET2 (QRT2), part of a small family of endo-PGs, is involved in the anther dehiscence process [4, 19]. Many papers have shown that the septum and stomium undergo a process of degeneration and cell death via a developmental programmed cell death (PCD)-related process, as part of dehiscence. Furthermore, plant cells usually undergo PCD in response to hormone-mediated signalling pathways $[8,20]$. Among the genes associated with PCD, in $A$. thaliana, PROMOTION OF CELL SURVIVAL1 (PCS1) has been recognized as an important factor encoding an aspartic protease that acts during reproduction and embryogenesis. Using transgenic plants that overexpressed PCS1, Ge et al. showed that this gene inhibits the individual elements of the PCD pathway in anthers [21].

Examination of late-dehiscence mutants revealed that the time of anther opening is strictly controlled by various factors, including phytohormones. Usually, A. thaliana mutants that are defective in phytohormone biosynthesis or perception are male sterile and have anthers that split too late for effective pollination and fertilization [8, 9, 22, 23]. Gibberellins (GAs) play an important role in the control of pollen formation and viability, filament elongation and anther dehiscence [5, 2428]. Late GA biosynthesis stages are catalysed by gibberellin 3-oxidases (GA3oxs) responsible for the production of active phytohormone molecules and gibberellin 2oxidases (GA2oxs) that deactivate GAs. The central factor in the GA signalling pathway is GAMYB, which belongs to the R2R3-MYB subfamily. The results of research on barley (Hordeum vulgare) show that overexpressing $H \nu G A M Y B$ results in a phenotype with indehiscent anthers [29]. Another study revealed that GAMYB acts in cooperation with microRNAs, especially miR159 [30]. These facts indicate that a complex set of interactions is required to coordinate events within the flower that lead to anther dehiscence.

The developmental timing of anther opening influences the type of pollination and degree of selfpollination in comparison with cross-pollination. This is important for seed production in breeding programs and agriculture. In general, pollen release occurs when flowers are fully open, in A. thaliana at the flower stage 13 [31]; however, in some plants, including yellow lupine (Lupinus luteus L.), a special type of self-pollination in a closed flower (cleistogamy) was found. Yellow lupine is also characterized by the ability to fix atmospheric nitrogen, and high protein content in seeds; therefore, it is used worldwid as a food and feed source. Nevertheless, this legume plant has the problem of premature flower abscission [32-35]. For years, there has been a 
hypothesis that the reason for excessive flower abscission may be incorrect stamen development and, as a consequence, insufficient pollination, fertilization, and pod setting, which reduce yield. Our earlier study showed that changing the level of LIDELLA1 mRNA, encoding major repressors of GA signalling, supports correct flower and pod development [34]. Interestingly, the expression of LIDELLA1 in the flower bud phase was relatively high and then decreased slightly until anther opening; during pollination, fertilization and early pod development, a gradual increase in transcript levels was observed. This indirectly indicates the involvement of GAs in flower development. In different plant species, research on the role of GAs in stamen development has focused on the early stages, and there are few or no available data on their effects in late stages, including anther dehiscence. Therefore, the main aim of this study was to understand the unexplored process of GAmodulated late anther development in yellow lupine, which may lead to the control of male fertility in the future and aid in eliminating the problem of low yield. We focused on genes and proteins that could be potential markers of developmental changes taking place in yellow lupine anthers. Examination of the expression profile of genes widely associated with dehiscence after the application of various compounds (gibberellic acid, $\mathrm{GA}_{3}$ and paclobutrazol, $\mathrm{PAC}$ ) in conjunction with $\mathrm{GA}_{3}$ immunolocalization provides broad insight into the regulation of the anther development process in yellow lupine. The function of predicted proteins was defined based on the presence of conserved domains, motifs and specific amino acids. The research is complemented by histological analyses to determine the changes in the structure of the yellow lupine anther during the process of extensive dehiscence.

\section{Results \\ Specific changes in structure of yellow lupine anthers in different stages of late development}

The individual stages of yellow lupine late anther development (LAD), when dehiscence takes places, were selected (Fig. 1A). In the first phase of LAD (1 LAD), which corresponds to $A$. thaliana phase 11, four separated locules with pollen were distinguished (Fig. 1B). Therefore, the anther is bithecal and tetrasporangiate. In the centre of the yellow lupine anther, connective tissue containing a vascular bundle with a septum on both sides (which separate the locules) was found. Moreover, septum cells are characterized by different sizes depending on their location, with a tendency that cells closer to the anther centre are much larger. The wall surrounding the anther locules consists of the following cell layers: epidermis, endothecium, middle layer, and tapetum. The externally located epidermis is composed of one layer of longitudinally elongated cells. The differentiated epidermal cells form the stomium region adjacent to septum cells. The next layer consists of transversely elongated endothecial cells with visible secondary cell wall thickening. Additionally, the remains of the tapetum were visible in locules (Fig. 1B). In the 1 LAD phase, breakage of the septum from the stomium was also noticed (Fig. 1C). This generates a bilocular anther, and further degeneration of the septum cells was evident in the second LAD phase (2 LAD) (corresponding to A. thaliana phase 12) (Fig. 1D). The stomium forms a single-cell region, the location of which determines the position of anther opening. Late septum shrinkage and disruption seem to contribute to breaking the stomium, which leads to pollen release (these processes occur simultaneously). The dehiscence process does not affect the central connective cells, which remain joined. Yellow lupine anther opening occurs in 2 LAD, which corresponds to phase 13 in A. thaliana (Fig. 1E). Following dehiscence, anther senescence occurs (3 LAD, Fig. 1F/G), characterized by further degeneration and shrinkage of cells, and the entire anther structure (4 LAD, Fig. 1H/I).

\section{Secondary thickening of endothecial cell walls}

In the first stage of yellow lupine anther dehiscence, the endothecium, which partially surrounds the locule, undergoes the formation and deposition of secondary thickening (Fig. 2A). The endothecial cell walls show Ushaped thickening. The same occurs in the connective cell walls but to a much lesser extent. Other anther cells, which create, for example, epidermis, septum or stomium region, do not undergo thickening, which suggests that areas of secondary thickening are strictly localized in the endothecium.

The expression profiles of secondary wall-associated genes (Fig. $2 \mathrm{~B}$ ) with and without $\mathrm{GA}_{3}$ application in all LAD phases in yellow lupine were established (Fig. 2C). In the first and second LAD phases, significantly higher expression levels of $L l C A D$ and all LlIRX genes were observed compared to those in the third and fourth LAD phases. In addition, $\mathrm{GA}_{3}$ treatment increased the number of lignin/cellulose/xylan biosynthesis gene transcripts, especially in the second LAD phase. It can be concluded that the genes related to secondary thickening of the endothecial cell walls show co-upregulated expression, and their transcriptional activity is mainly associated with changes in the first and second LAD stages of yellow lupine. Additionally, it appears that the levels of transcripts of all identified genes are GA dependent.

\section{Breakdown of the septum/stomium cells}

Cell separation is an important event that takes place in yellow lupine anthers. Dehiscence involves interruption of the cell wall material between adjacent 


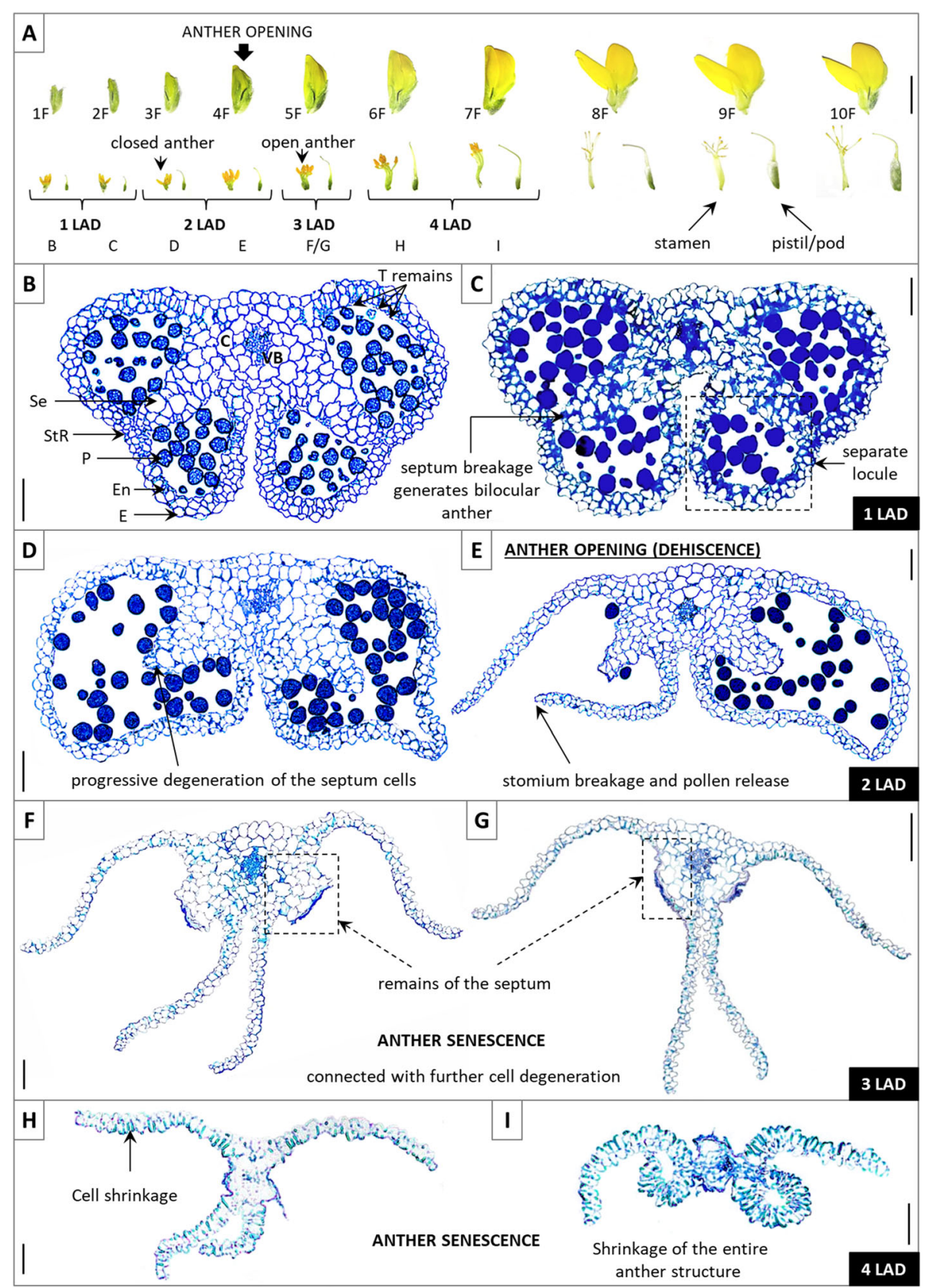

Fig. 1 A Individual stages of flower ( $1 \mathrm{~F}-10 \mathrm{~F})$ development in yellow lupine. After completing the dehiscence (1F-3F), anther opening occurred at approximately the fourth phase of flower development (4F) when the flower was completely closed. Then, pollination, fertilization, and pod setting and development occurred (5F-10F). The first and second stages of flower development correspond to the first stage of late anther development (LAD); the third and fourth stages of flower development correspond to the second stage of LAD; the fifth stage of flower development is parallel to the third stage of LAD; and the sixth and seventh stages of flower development correspond to the fourth stage of LAD. B-I The anatomical structure of yellow lupine anthers in different stages of late development. Cross-sections were stained with toluidine blue, and anthers were photographed by light microscopy. C - connective, VB - vascular bundle, Se - septum, StR - stomium region, P - pollen grain, En - endothecium, E - epidermis, T - tapetum. Scale bars = $1 \mathrm{~cm}(\mathrm{~A}) ; 50 \mu \mathrm{m}(\mathrm{B}-\mathrm{I})$

cells (Fig. 3A). It is likely that endo-PGs and phytohormones are involved in this process. In selected stages of LAD, we examined the transcriptional activity of $L l Q R T 2$, encoding the marker enzyme involved in breakdown of pectin between cells (Fig. 3B). The results indicate that the amount of LlQRT2 mRNA is high in the first and second LAD phases, when the breakdown of the septum/stomium takes place. Lower levels of transcripts were detected in the third and fourth LAD phases. Interestingly, LlQRT2 expression 

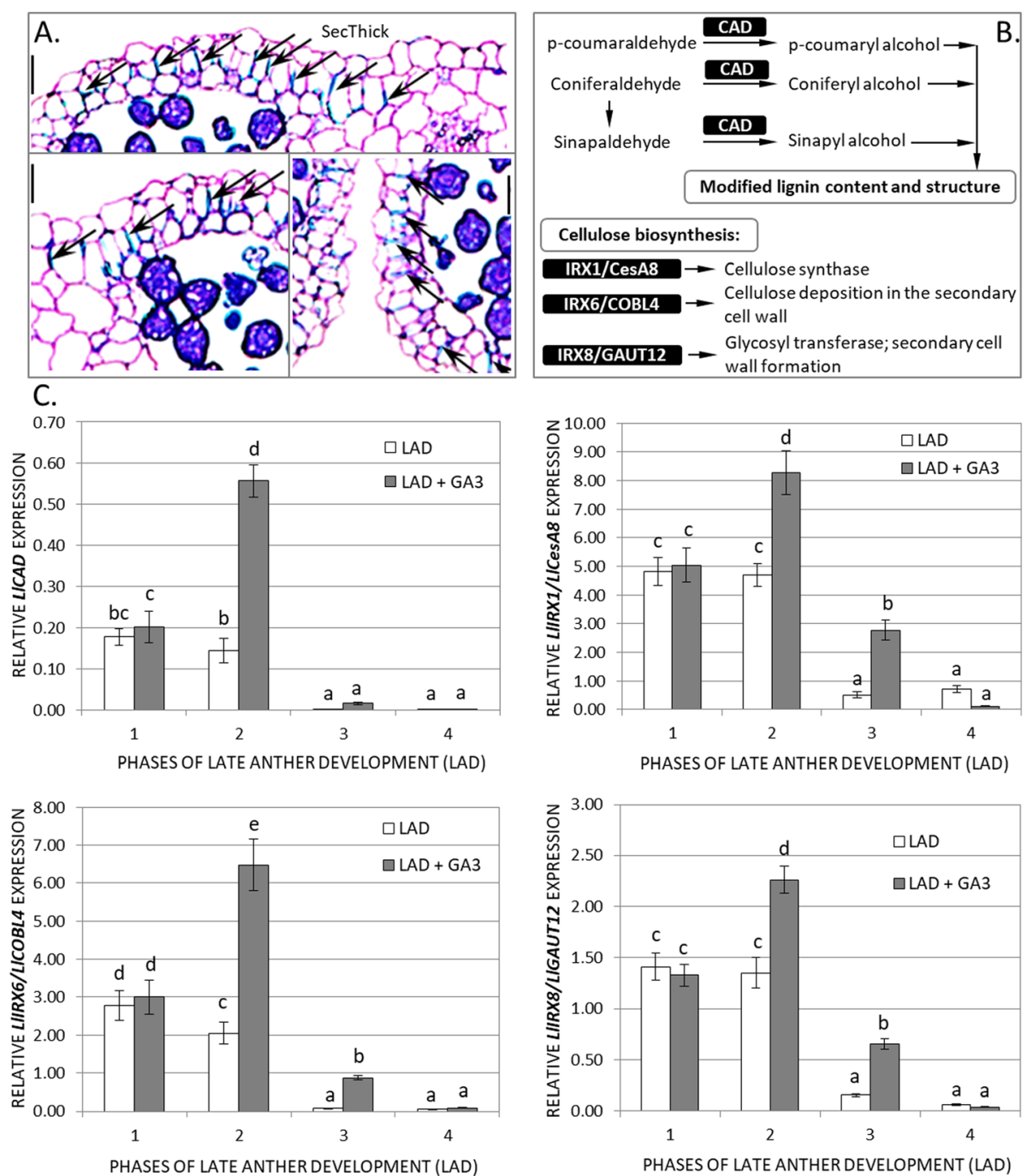

Fig. 2 A Localization of secondary thickening (SecThick) within endothecial cell walls of yellow lupine anthers. Transverse sections were stained with toluidine blue. Anthers were photographed by light microscopy. B The function of typical lignin (CAD, CINNAMYL ALCOHOL DEHY DROGENASE) and cellulose (IRX1/CesA8, IRREGULAR XYLEM1/Cellulose synthase A catalytic subunit 8; IRX6/COBL4, COBRA-like4; IRX8/GAUT12, GAlactUronosylTransferase 12) biosynthesis-related genes [15, 18, 36, 37]. C Transcriptional activity of investigated genes (related to LIACT) during late anther development (LAD) and after gibberellic acid treatment (LAD $\left.+G A_{3}, 100 \mu \mathrm{M}\right)$. Data are the mean $\pm S E$ of three biological replicates, each with two technical replicates. Letters represent statistically significant differences at $p<0.05$ (one-way ANOVA followed by Tukey's honest significant difference test). Scale bars $=25 \mu \mathrm{m}$

was stimulated by $\mathrm{GA}_{3}$, especially in the second $\mathrm{LAD}$ stage.

\section{Degeneration of septum/stomium cells via PCD-related processes}

The yellow lupine anther septum and stomium undergo degeneration and cell death to facilitate pollen release via GA-dependent PCD-related processes (Fig. 3C). To prove this, we determined the expression profile of LlPCS1, which encodes aspartic protease and is an anticell death component. As expected, $\mathrm{GA}_{3}$ application decreased the transcriptional activity of LlPCS1, especially in the first and fourth stages of LAD (Fig. 3D). This indirectly shows that $\mathrm{GA}_{3}$ favours this process in yellow lupine anthers by inhibiting the anti-PCD factor.

\section{Cellular and tissue localization of gibberellic acid during yellow lupine anther development}

We performed cellular and tissue immunolocalization of $\mathrm{GA}_{3}$ in all selected stages of anther maturation in yellow lupine (Fig. 4). The results show that the highest accumulation of $\mathrm{GA}_{3}$ occurred in the first LAD phase before anther opening (Fig. 4A). At the cellular level, the strongest fluorescence signal was noted in the entire cytoplasm of degenerating septum cells at the time of bilocular anther formation (the cells were filled with 


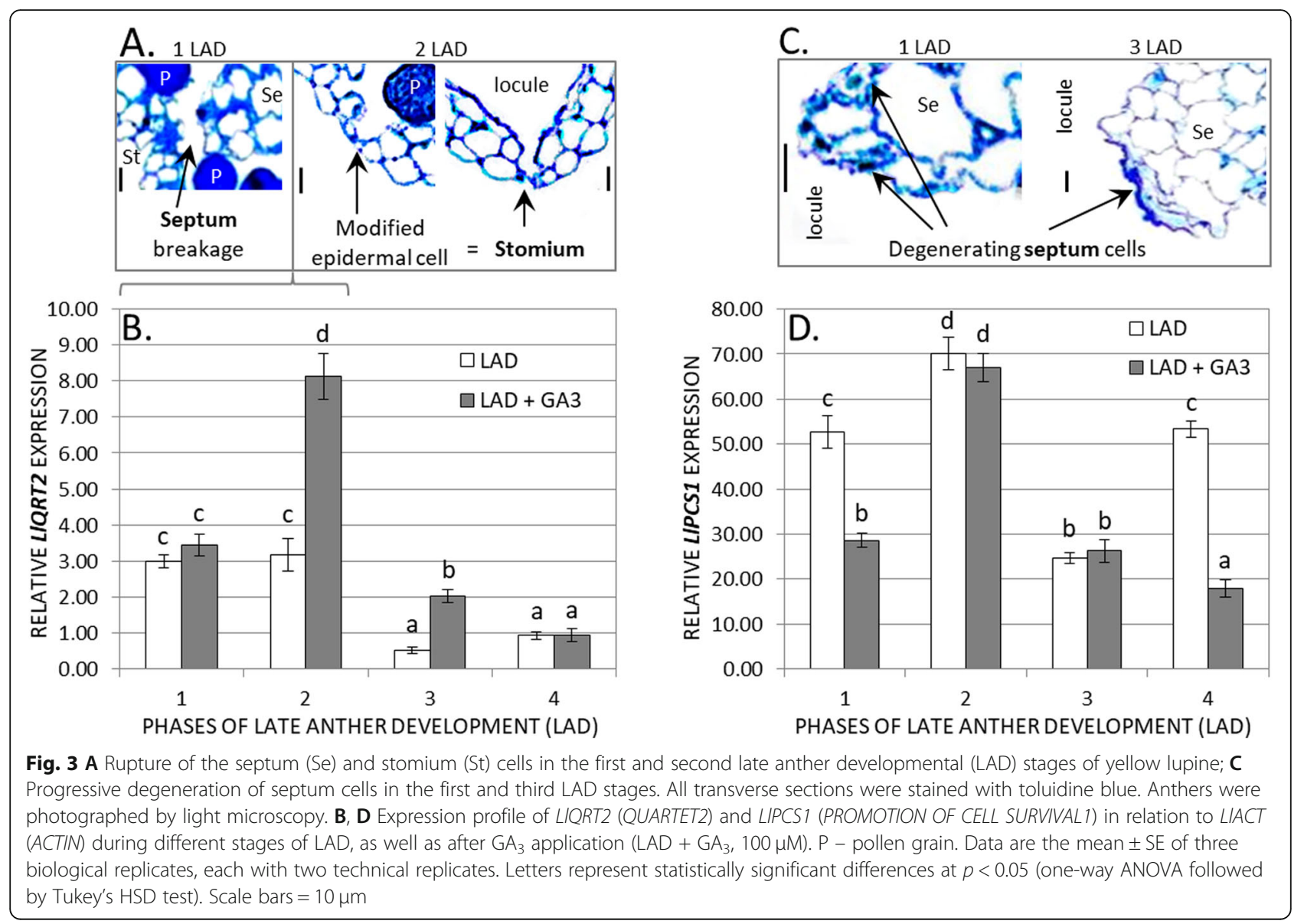

$\mathrm{GA}_{3}$; Fig. 4A1). A high level of phytohormone molecules was also observed near the vascular bundle, in the cells of the middle layer and endothecium, and in the epidermis to a lesser extent. In the second LAD phase, when the stomium cells were disrupted and the pollen chambers opened, the fluorescence signal indicating $\mathrm{GA}_{3}$ content was almost imperceptible (Fig. 4B). GA 3 was found in the cells of the progressively degenerating septum, middle layer, and near the vascular bundle (Fig. 4B1). After the anther was opened and mature pollen grains were released (3 LAD stage), no fluorescence signal indicating the presence of $\mathrm{GA}_{3}$ was observed (Fig. $4 \mathrm{C}$ ). $\mathrm{GA}_{3}$ signal was also not detected when complete cell degeneration and ageing of the anther occurred (4 LAD stage). The lack of cell nuclei was also noticeable, which indicates the rapidly progressing processes of degeneration and death of entire cells of the anther (Fig. 4D).

\section{$\mathrm{GA}_{3}$ localization correlates with $\mathrm{GA}$ metabolism}

The expression profiles of $L l G A 3 o x$ involved in the formation of active phytohormone molecules and LlGA2ox1 responsible for GA inactivation were determined (Fig. 5) to verify whether they are correlated with the endogenous $\mathrm{GA}_{3}$ level. In subsequent stages of LAD, a decrease in
LlGA3ox mRNA levels was observed (Fig. 5A). In the case of LlGA2oxl, the opposite situation was found (Fig. 5B). Furthermore, the use of PAC, which inhibits the early stage of GA biosynthesis, significantly reduced the expression of both studied genes.

\section{$\mathrm{GA}_{3}$ controls LIGAMYB expression via a miR159- dependent pathway during late anther development} In yellow lupine, $\mathrm{GA}_{3}$ likely participates in the regulation of $L l G A M Y B$ by controlling the LlMIR159 expression level. The LlGAMYB and LlMIR159 transcriptional activity in four selected phases of LAD, as well as after $\mathrm{GA}_{3}$ and PAC application, were examined (Fig. 6). The $L l G A M Y B$ expression was almost identical with or without $\mathrm{GA}_{3}$ treatment, but the lack of $\mathrm{GA}_{3}$ due to PAC application resulted in increased $L l G A M Y B$ transcripts, especially in the first and second LAD stages (Fig. 6A). These results suggest that $\mathrm{GA}_{3}$ possibly indirectly regulates $L l G A M Y B$ expression. Therefore, we examined the LlMIR159 expression profile (Fig. 6B). GA $\mathrm{A}_{3}$ treatment increased LIMIR159 mRNA levels, and importantly, PAC application significantly decreased the expression of LlMIR159. By analysing the natural conditions without the application of any compounds, it can be concluded 


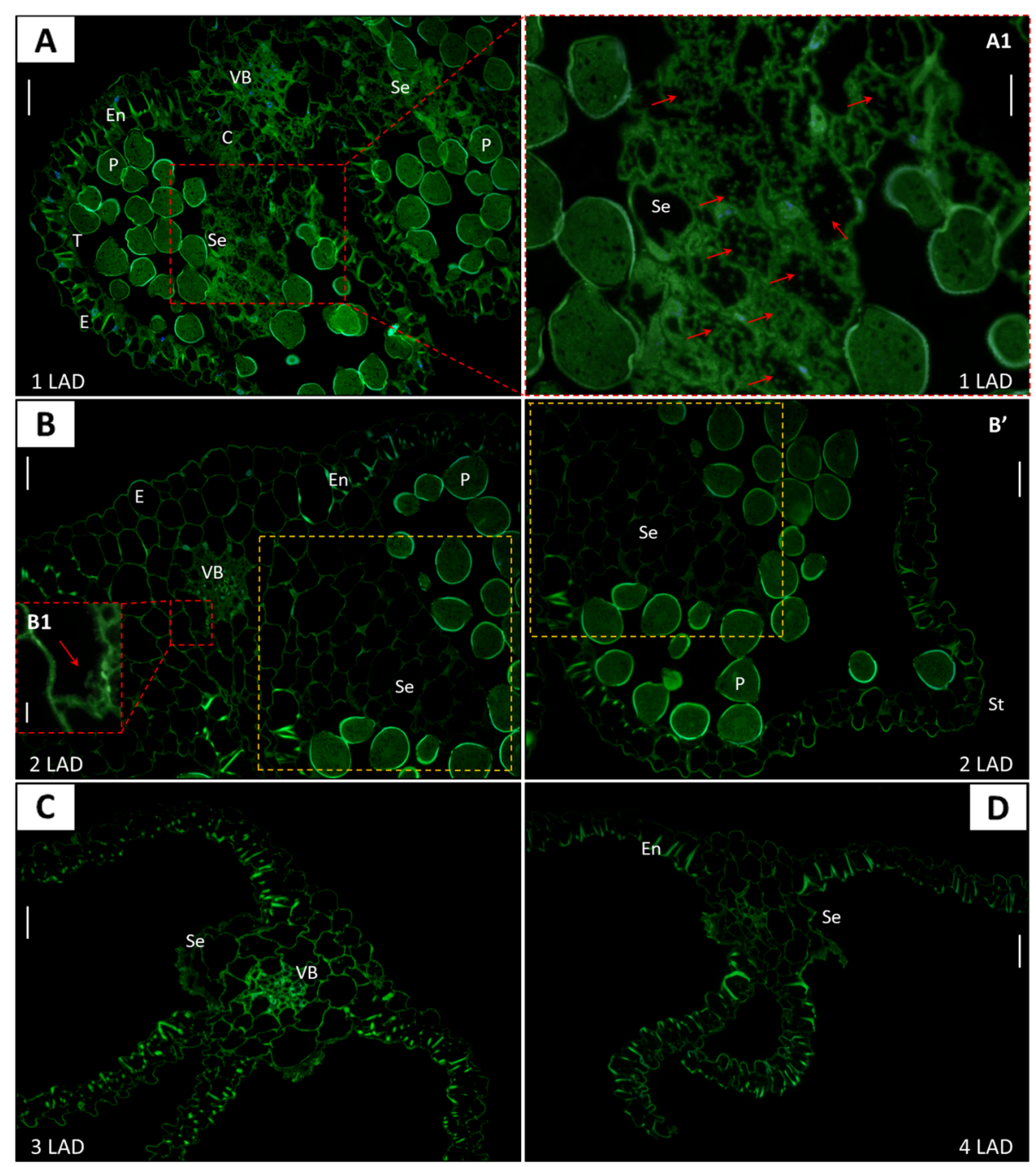

Fig. 4 Gibberellic acid $\left(G A_{3}\right)$ immunolocalization in selected stages (1-4) of late anther development $(L A D)$ in yellow lupine. Green fluorescence corresponds to $\mathrm{GA}_{3}$ accumulation, and blue fluorescence indicates cell nuclei stained with DAPI. Subfigures A1/B1 are an enlargement of subfigures $A / B$, respectively, in the places marked with the red squares. The red arrows indicate $G A_{3}$ signal in the selected magnified cells. The yellow squares marked with a dashed line in subfigures $B$ and $B^{\prime}$ indicate the same area of cells. Autofluorescence of the cell walls and pollen grains was visible. VB - vascular bundle, P - pollen grain, E - epidermis, En - endothecium, T - tapetum, C - connective, Se - septum, St stomium. Scale bars: $25 \mu \mathrm{m}\left(\mathrm{A}, \mathrm{B}, \mathrm{B}^{\prime}, \mathrm{C}, \mathrm{D}\right), 10 \mu \mathrm{m}$ (A1) and $5 \mu \mathrm{m}$ (B1)

that $L l G A M Y B$ expression is high in the first two stages of LAD and then decreases. This negatively correlates with the transcriptional activity of LIMIR159, which was significantly increased in the second phase of LAD. This is probably the cause of the reduced mRNA content of LIGAMYB in the third and fourth LAD stages. The results suggest that $L l G A M Y B$ is coexpressed with LIMIR159 in yellow lupine anthers. It also follows that the $L l G A M Y B$ transcript level is potentially regulated by miR159 in the anthers of yellow lupine.

\section{In silico analyses of studied genes}

The identification of many genes encoding enzymes connected with anther dehiscence prompted us to perform bioinformatics analyses. The full-length cDNA sequences identified in yellow lupine, their deduced amino acid sequences, molecular weights, predicted isoelectric points and NCBI accession numbers are presented (Fig. S1a, S2a, S3a, S4a, S5a, S6a, S7a, S8a, S9a). Maximum likelihood phylogenetic trees of different proteins with the highest degrees of similarity/identity to proteins predicted in yellow lupine were constructed (Fig. S1b, S2b, S3b, S4b, S5b, S6b, S7b, S8b, S9b). In all cases, the yellow lupine proteins showed the closest relationship to proteins derived from legumes, especially narrow-leaf lupine ( $L$. angustifolius). To better understand the function of all yellow lupine proteins, the conserved domains, motifs and specific amino acids were 

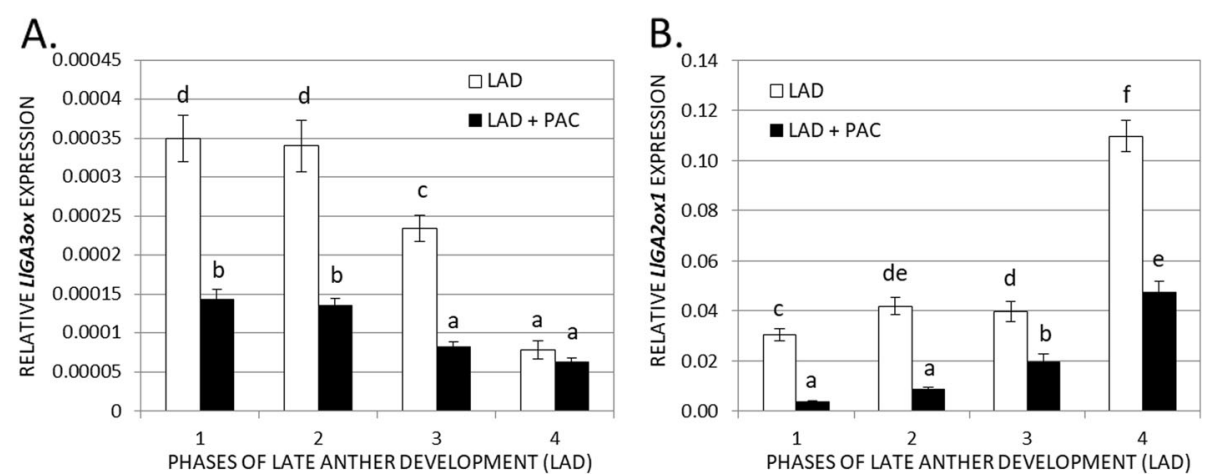

Fig. 5 The relative transcript levels of LIGA3ox (gibberellin 3-oxidase) connected with GA biosynthesis (A) and LIGA2ox1 (gibberellin 2-oxidase 1) involved in GA deactivation (B) were investigated during late anther development (LAD) of yellow lupine. Some anthers were treated with a solution of paclobutrazol (LAD + PAC, $100 \mu \mathrm{M})$ in 0.05\% Tween 20, and other anthers were treated with only 0.05\% Tween 20 (LAD). LIACT (ACTIN) was used as an internal control. Data are the mean \pm SE of three biological replicates, each with two technical replicates. Letters represent significant differences at $p<0.05$ (one-way ANOVA followed by Tukey's HSD test)

discovered and localized (Fig. S11A'-F'; Fig. S12A'-C'). The positions of all conserved domains, motifs and amino acids present in yellow lupine were determined and confirmed using the background of similar proteins occurring in many plant species (Fig. S1c, S2c, S3c, S4c, S5c, S6c, S7c, S8c, S9c). Additionally, the predicted yellow lupine proteins were assigned a likely function based on those described in other plant species (Table 1). The tertiary structures of yellow lupine proteins were predicted using the Robetta service (Fig. S11A-F; Fig. S12A-C). Additionally, the amino acid sequences of yellow lupine were compared with those of other plant species. Both the similarity and the number of identical amino acids were included (Fig. S1d, S2d, S3d, S4d, S5d, S6d, S7d, S8d, S9d). It follows that proteins such as CAD, CesA8, COBL4 or GAUT12 are strictly conserved among the plant kingdom, while the remaining proteins (PG/QRT2, PCS1, GA3ox, GA2ox and GAMYB) show a greater affinity among closely related species but much lower identity/similarity with species as $A$. thaliana. The complete cDNA sequence of Ll-MIR159 was identified in yellow lupine (Fig. S10) and compared with MIR159 sequences cloned in other plant species that showed the highest similarity. On this basis, a phylogenetic tree was constructed (Fig. 7A). Further Ll-MIR159 analyses revealed very high similarity of the 21-nt fragment that forms a mature miRNA to another identified in different plant species. In 11 of the 12 species compared, these fragments of sequences were $100 \%$ identical (Fig. 7B). The fragment of secondary stem-loop structure of Ll-pre-miR159 and localization of mature miR159 on the stem of the precursor sequence is presented (Fig. 7C). The alignment of the nucleotide sequences of Ll-pre-miR159 with mature miR159 in Manihot esculenta, Dimocarpus longan, Populus trichocarpa, Populus tomentosa, Cucumis melo, Citrus sinensis and A. thaliana is shown (Fig. 7D). The alignment of some nucleotide
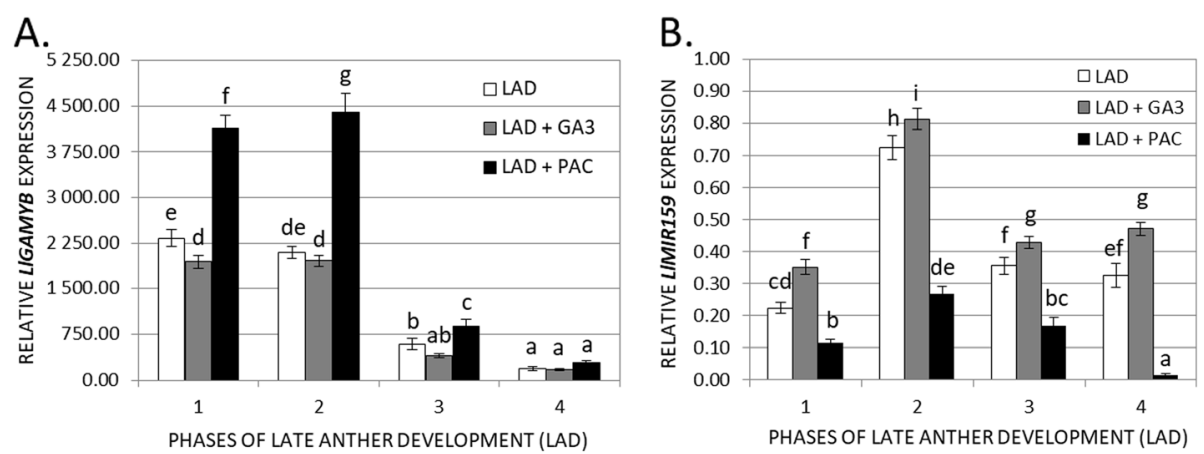

Fig. 6 Relative transcriptional activity of LIGAMYB involved in GA signalling (A) and LIMIR159 associated with cutting GAMYB transcripts (B) during late anther development $(L A D)$ of yellow lupine. Some anthers were treated with a solution of $G A_{3}\left(L A D+G A_{3}, 100 \mu M\right)$ in $0.05 \%$ Tween 20 , others were treated with a solution of paclobutrazol (LAD + PAC, $100 \mu \mathrm{M})$ in 0.05\% Tween 20, and some were treated with only $0.05 \%$ Tween 20 (LAD). LIACT (ACTIN) was used as an internal control. Data are the mean \pm SE of three biological replicates, each with two technical replicates. Letters represent statistically significant differences at $p<0.05$ (one-way ANOVA followed by Tukey's HSD test) 
Table 1 Predicted function of conserved domains, motifs and specific amino acids in yellow lupine proteins [LICAD (cinnamyl alcohol dehydrogenase); LICesA8/LIIRX1 (cellulose synthase A catalytic subunit 8/IRREGULAR XYLEM1); LICOBL4/IRX6 (COBRA-like4); LIGAUT12/LIIRX8 (galacturonosyltransferase12); LIPG/LIQRT2 (polygalacturonase/ QUARTET2); LIPCS1 (PROMOTION OF CELL SURVIVAL1); LIGA30x (gibberellin 3-oxidase); LIGA20x1 (gibberellin 2-oxidase1); LIGAMYB)] based on data published in other plant species

\begin{tabular}{ll}
\hline Protein & Identified conserved domains/motifs/specific amino acids \\
\hline LICAD & Alcohol dehydrogenase GroES-like domain \\
& Zinc-binding dehydrogenase domain \\
& Zn-1 (GHExVGxVxxxGxxV) and \\
& Zn-2 (GxxVGxGxxxxxCxxCxxCxxxxxxxC) \\
& binding motifs \\
& Three amino acids C, H, C \\
& Four C residues \\
& G residues (GxGGxG) (so-called Rossmann fold) represent NADPH \\
& co-substrate-binding motif \\
& S 212 \\
& Many conserved residues: S, Q, L, M, W, V, P, L, F, I \\
& N-terminal region inclusive of a Zn-binding RING motif with a \\
& strictly conserved CxxC sequence motif beginning amino acids: \\
& CxxCx 12 FxACxxCxxPxCxxCxExxxxDDxxxCxxC \\
& Hypervariable region (VR1) of 117 aa, rich in acidic aa \\
LICesA8 &
\end{tabular}

Two transmembrane domains near the $\mathrm{N}$-terminus (TMH1-2) and six transmembrane domains (TMH3-8) at the C-terminus

Large cytosolic/catalytic central domain (CD = globular domain = soluble domain), which includes the Plant Conserved Region (PCR) within Conserved Region 1 (CR1), Class Specific Region (CSR) within Variable Region 2 (VR2) and Conserved region 2 (CR2)

Located in the CD domain A consists of several widely spaced aspartic acid (D) residues - a single D followed by a DxD

Located in the CD domain B consists of a third conserved aspartic acid (D) residue and three conserved amino acids QxxRW

LICOBL4/ The putative conserved domain characteristic to COBRA LIIRX6 superfamily

$\mathrm{N}$-terminal signal peptide with cleavage site

The putative cellulose-binding site

The central Cys-rich (CCVS) motif

Two conserved consensus N-glycosylation sites

Locus corresponds to the predicted cleavage $\omega$-site at the Cterminus

LIGAUT12/ N-terminal cytoplasmic domain

LIIRX8

The transmembrane domain

The specific glycosyl transferase family 8 (GT8) domain

The catalytic DxD motif

Four typical conserved domains I, II, III and IV

LIORT

Three aspartic acids (D) in domains I and II

The histidine residue $(\mathrm{H})$ in domain III

Predicted functions

Catalytic domain with GroES-like structure [38]

Catalytic activity, zinc ion binding [38]

Zn-1 catalytic centre and

$\mathrm{Zn}-2$ binding site [39]

Define places of catalytic $\mathrm{Zn}$ action [40]

Structural Zn ligation (Zn-2 structural motif) [40]

$G$ residues for substrate specificity [40]

Specific NADP(H) binding residue [40]

Determine substrate ligation [38]

Protein-protein interactions in the CesA complex [41-43]

This region is more conserved than was previously thought. The contribution of this region to the overall function of the enzyme is unknown $[41,43]$

Transmembrane helixes [44]

[41-43]

These residues bind the UDP-glucose substrate. Processive enzymes catalyse the addition of many sugar residues to a growing chain $[41,43]$

Part of the catalytic site $[41,43]$

\section{$\mathrm{CDD}(\mathrm{NCBI})$}

Signal peptide cleavage site $[45,46]$

A carbohydrate-binding module (CBM) [46]

Highly conserved and characteristic for all COBL proteins $[45,46]$

Asparagine (N)-linked glycosylation of protein [46]

Glycosylphosphatidylinositol (GPI) modification motif. GPI anchors are added through an amide bond onto the last amino acid residue remaining after cleavage of the $\omega$-site [46]

Phyre $^{2}$

Phyre $^{2}$

Transfer sugar residues to donor molecules. CDD (NCBI)

\section{CDD (NCBI)}

The well-conserved positively charged domain IV (RIKT) constitutes a likely candidate for ionic interactions with carboxylate groups present in the substrate [47-49]

The carboxylate group in aspartic acids in NTD and DD structures (domains I and II, respectively) may be a component of the catalytic site [50]

Participates in catalytic reaction [51]

A tyrosine $(Y)$ at position 320 
Table 1 Predicted function of conserved domains, motifs and specific amino acids in yellow lupine proteins [LICAD (cinnamyl alcohol dehydrogenase); LICesA8/LIIRX1 (cellulose synthase A catalytic subunit 8/RREGULAR XYLEM1); LICOBL4/RX6 (COBRA-like4); LIGAUT12/LIIRX8 (galacturonosyltransferase12); LIPG/LIQRT2 (polygalacturonase/ QUARTET2); LIPCS1 (PROMOTION OF CELL SURVIVAL1); LIGA30x (gibberellin 3-oxidase); LIGA20x1 (gibberellin 2-oxidase1); LIGAMYB)] based on data published in other plant species (Continued)

\begin{tabular}{|c|c|c|}
\hline Protein & Identified conserved domains/motifs/specific amino acids & Predicted functions \\
\hline & 12 cysteine $(C)$ residues & $\begin{array}{l}\text { Important to maintain the three-dimensional structure of extracellular } \\
\text { proteins and are distributed all along the sequences but with a higher } \\
\text { frequency at the C-terminal end [49] }\end{array}$ \\
\hline \multirow[t]{5}{*}{ LIPCS1 } & $\begin{array}{l}\text { Two motifs in both N (DTGS) and C (DS/LGT)-terminal ends } \\
\text { characteristic for pepsin like aspartic proteases }\end{array}$ & Catalytic motifs (CDD, NCBI) \\
\hline & Two catalytic residues (D) & $\begin{array}{l}\text { Plays key catalytic roles in the pepsin family and conserved for all } \\
\text { family members (CDD, NCBI) }\end{array}$ \\
\hline & Active site flap ATLS and SSSS & $\begin{array}{l}\text { An extended loop projecting over the cleft to form an 11-residue flap, } \\
\text { which encloses substrates or inhibitors within the active site. It also } \\
\text { contributes three residues for substrate specificity (CDD, NCBI) }\end{array}$ \\
\hline & Pepsin A like plant domain & $\begin{array}{l}\text { Characteristic for chloroplast nucleoids DNA-binding protease and } \\
\text { nucellin, pepsin-like aspartic proteases (CDD, NCBI) }\end{array}$ \\
\hline & $\begin{array}{l}\text { TAXi_N domain; TAXI_C domain } \\
\text { Xylanase inhibitor }\end{array}$ & $\begin{array}{l}\text { The } \mathrm{N} \text { - and C-termini of the members of this family are jointly neces- } \\
\text { sary for creating the catalytic pocket necessary for cleaving xylanase } \\
\text { (cell-survival processes) (CDD, NCBI) }\end{array}$ \\
\hline \multirow[t]{3}{*}{ LIGA3ox } & Gibberellin 3- $\beta$-dioxygenase domain & $\mathrm{CDD}(\mathrm{NCBI})[53]$ \\
\hline & $\begin{array}{l}\text { 2-oxoglutarate (2OG) and Fe (II)-dependent oxygenase (Oxy) } \\
\text { superfamily domain }\end{array}$ & $\mathrm{CDD}(\mathrm{NCBI})$ \\
\hline & The His-x-Asp-(x)n-His (HxD ...H) and Arg-x-Ser (RxS) motifs & Recruit Fe (II) as a cofactor and co-substrate CDD (NCBI) \\
\hline \multirow[t]{3}{*}{ LIGA20x1 } & Gibberellin 2- $\beta$-dioxygenase domain & $\mathrm{CDD}(\mathrm{NCBI})$ \\
\hline & $\begin{array}{l}\text { Domain characteristic for 2-oxoglutarate (2OG)-Fe (II)-dependent } \\
\text { oxygenase superfamily }\end{array}$ & $\mathrm{CDD}(\mathrm{NCBI})$ \\
\hline & The $H \times D \ldots H$ and $R \times S$ motifs & $\begin{array}{l}\text { Amino acid residues presumed to bind } \mathrm{Fe}^{2+} \text { at the active site of } \\
\text { protein }\end{array}$ \\
\hline \multirow[t]{4}{*}{ LIGAMYB } & R2R3 domain & Near the $5^{\prime}$ terminus \\
\hline & Box 1, Box 2, Box 3 domains & Distributed throughout the protein \\
\hline & REB1 domain & $\begin{array}{l}\text { Characteristic for Myb superfamily proteins, including transcription } \\
\text { factors and mRNA splicing factors }\end{array}$ \\
\hline & $\begin{array}{l}\text { Myb_DNA-binding domain and SANT (SWI3, ADA2, N-CoR and } \\
\text { TFIII') domains }\end{array}$ & DNA-binding domains have been designated using CDD (NCBI) \\
\hline
\end{tabular}

sequences of LlGAMYB (Ll-miR159 target gene) with homologous genes in Glycine soja, G. max and $A$. thaliana is also presented (Fig. 7E).

\section{Discussion}

\section{Changes in anther structure of cleistogamous yellow lupine}

The dehiscence of anthers is a multistage process that has been examined in species such as A. thaliana, rice, maize (Zea mays), tobacco and tomato (Solanum lycopersicum) [4]. In our previous study, we showed that yellow lupine anther opening takes place in the fourth stage of flower development [34]. Therefore, it is relevant to study the process of late anther development and accompanying alterations in the tested cleistogamous species. In this paper, we established that the anther wall consists of epidermis, endothecium, middle layers and a tapetum, similar to its composition in other plant species, including both cleistogamous species, such as barley and wheat (Triticum L.), and chasmogamous species, such as tomato and rice [10, 54-56]. The yellow lupine epidermis protects all anther tissues and develops the stomium, which acts as the site for anther opening, whereas modifications of the endothecium play a critical role in pollen release. The other anther tissues in yellow lupine act similarly to several plant species; hence, it follows that the anther dehiscence process is widely preserved $[1,4,8,10,54,57]$. Nevertheless, there are certain exceptions [12]. Unlike yellow lupine, where the processes of stomium breakage and anther opening are connected in time, in some species such as Allium triquetrum, these processes are separated. After the rupture of both stomium simultaneously, each $A$. triquetrum whorl does not fully open, and pollen is not released due to unbroken epidermal cells [58]. Legumes are one of the largest families of cleistogamous plants, although there are different types of cleistogamy [59]. Lupine species show often pre-anthesis cleistogamy 


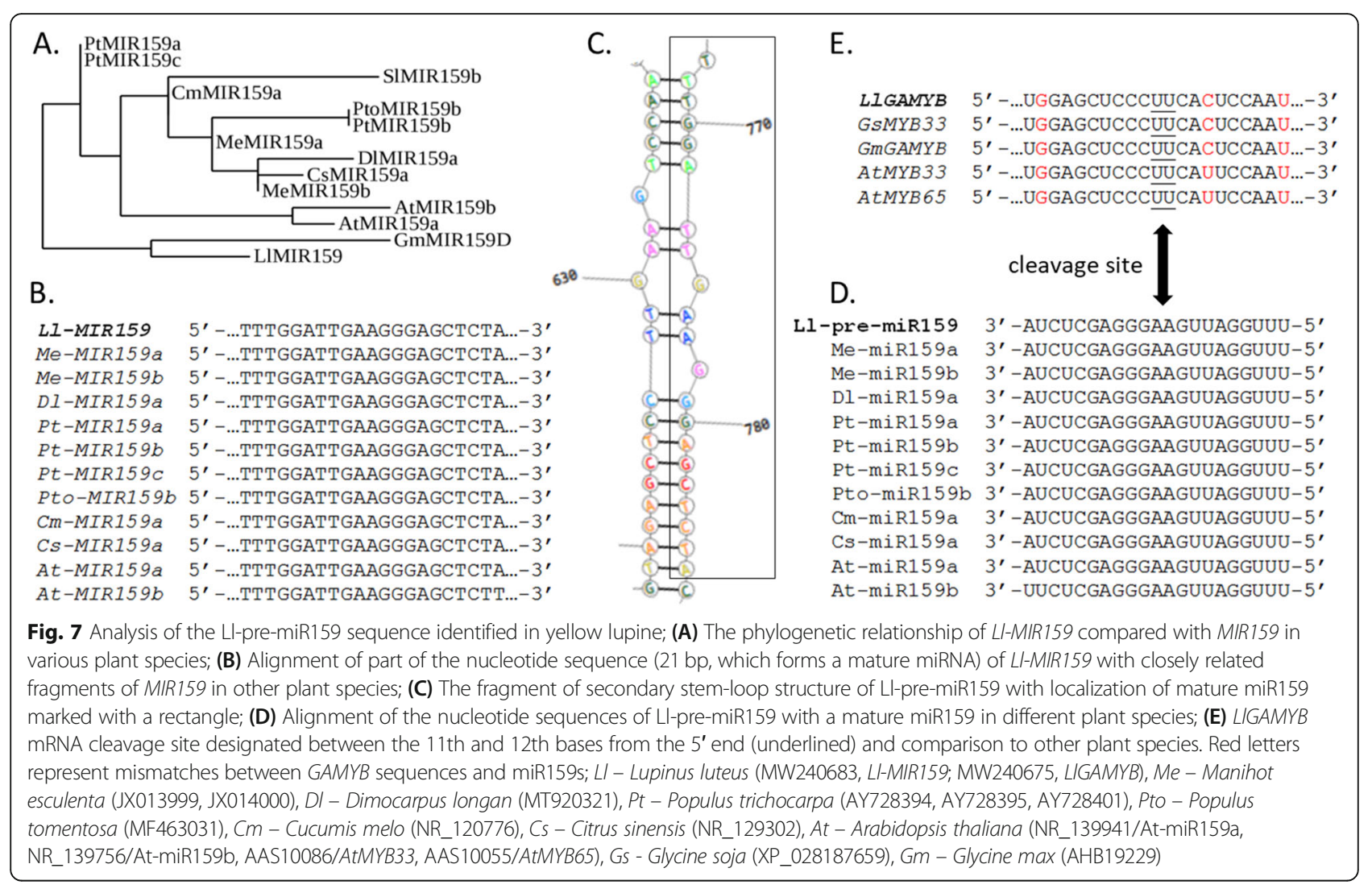

when pollination occurs during the bud stage, but the flower ultimately opens. Most annual lupine species reproduce via self-pollination, but cross-pollination is also possible to a lesser degree [60]. In the case of peanut (Arachis hypogaea), there is dimorphic/true cleistogamy consisting of flower dimorphism and different developmental pathways within one individual or species [59, 61]. There are also species with only cleistogamous flowers (complete cleistogamy), including many orchids and grasses [59].

\section{The yellow lupine anther endothecium undergoes} lignocellulosic deposition leading to secondary thickening The secondary thickening of endothecial cell walls occurs in four basic forms depending on the species: (I) annular ribs, (II) helical ribs forming a pattern of Ushaped thickening, (III) reticulate ribs and (IV) palmate ribs $[4,11]$. Some species may have two types of thickenings, but the walls of the endothecium typically show only one type [11]. For example, in A. triquetrum there are helical and U-shaped thickening whereas in Crocus (Iridaceae) species the thickenings are modified in the form of repeated continuous rings [58, 62]. The location and shape of the endothecium can also vary in a speciesspecific manner. In yellow lupine, the endothecium partially surrounds the locule; the endothecium is located in the upper third of the anther in tomato [54] and the endothecium adheres circularly to the locule in maize [4, 63]. Additionally, in yellow lupine, secondary thickenings are mainly found in endothecial cells (much less connective) whereas in certain species, secondary thickenings are also found in other types of anther cells. $A$. triquetrum has thickenings in the endothecium as well as in the cells of the septum and the connective tissue surrounding the locules [58]. In some species, the thickening epidermis may perform a function similar to that of the endothecium, thereby providing mechanical force to support anther opening [64]. It is also known that dehiscence mechanisms can be variable, both among families and within a given family. The opening of the anther may be caused via cell lysis and/or mechanical force; with the aid of secondary cell wall thickening and desiccation, the stomium ruptures, releasing pollen grains.

The presence of lignin in the endothecium layer determines the formation of secondary cell wall thickening. In most species, the CAD enzyme is involved in the biosynthesis of lignin monomers, and converts cinnamyl aldehydes into their corresponding alcohols. The $A$. thaliana mutant lacking $C A D$ displays male sterility and, compared with wild-type (WT) plants, the lignin content is reduced and the lignin structure is greatly disrupted 
[14]. These changes cause a sterile mutant due to the lack of lignification of the endothecium, which does not result in secondary thickenings and hence does not release pollen. In our paper, we examined the transcriptional activity of $L l C A D$ during the late development of yellow lupine anthers. The highest level of $L l C A D$ expression was found in the first and second phases during which the process of endothecial cell wall lignification take place. Approximately 20 times more gene transcripts were found compared to the third and fourth LAD stages. This suggests that LlCAD participates in lignin biosynthesis in the tissues of yellow lupine anthers.

In addition to lignification of the endothecium, cellulosic thickening is also essential for anther dehiscence. The mutation of the A. thaliana CesA8/IRX1 gene responsible for the synthesis of cellulose, does not affect the formation of primary cell wall thickening, which allows for us to use this gene as a specific marker for secondary thickening $[15,16]$. In yellow lupine, the expression of LlIRX1/LlCesA8 was very high in the first two stages of LAD. This closely correlates with the transcriptional activity of $L l C A D$ and other identified genes - LIIRX6/LICOBL4 and LlIRX8/ LlGAUT12. In general, COBL genes, which encode plant-specific glycosylphosphatidylinositol (GPI) anchored proteins, are key regulators in the deposition of cellulose in the secondary cell wall [65]. In turn, xylan- and pectin-deficient irx8 has a mutated GAUT12 gene, the loss of which results in reduction of G lignin, changes in its deposition, and a lack of anther splitting [18].

Different phytohormones control the formation and deposition of secondary thickening in many plant species. For example, cytokinins (CKs) have been implicated in the regulation of secondary wall formation, since AHP4 (Arabidopsis histidine-containing phosphotransfer 4 ), which is an element of CK signalling, negatively regulates thickening in the endothecium [66]. Additionally, the transcriptional activity of AHP4 corresponds to IRX1/IRX6/IRX8 expression, which indicates that CKs control cellulose biosynthesis $[4,66]$. To date, little is known about the effect of GAs on the formation of secondary thickening; therefore, it became the subject of our research. The impact of this phytohormone treatment on the expression of all secondary thickeningrelated genes was very similar and consisted of increasing the number of transcripts, especially in the second and third LAD phases. In Cucumis melo, several hormone-responsive cis-regulatory elements in the $C A D$ promoter region were identified, including GAREs, TATC boxes and P-boxes characteristic of GAs [40]. It also confirms the important role played by GAs in the comprehensive progression leading to anther opening.

\section{The septum/stomium is enzymatically lysed and} undergoes PCD-mediated degeneration

In yellow lupine anthers, separation of the septum from the stomium cells seems to occur by a mechanism similar to the organ abscission process, which involves enzymatic lysis of the middle lamellae between the cells without their damage [35]. In our study, the expression profile of the $L l Q R T 2$ gene encoding a marker enzyme involved in pectin breakdown between cells during LAD in yellow lupine was investigated. The transcript level was highest when the septum and stomium split (first and second LAD phases, respectively). If the dissociation is mechanical, e.g., by stretching of expanding anther walls, it damages the cells involved [62]. Ogawa et al. (2009) have shown that the regulation of cell separation events involves combinations of PGs and phytohormones [19]. A. thaliana and tomato $p g$ mutants show delayed or blocked anther dehiscence; hence, it is known that the role of cell wall enzymatic lysis is important $[19,67]$. Additionally, the transcriptional activity of $A$. thaliana $P G$ - QRT2 is regulated by JA, ET and ABA [19]. In yellow lupine, it has been revealed that $\mathrm{GA}_{3}$ and the ET precursor regulate the functioning of the flower abscission zone; however, $\mathrm{GA}_{3}$ acts independently of $\mathrm{ABA}$ in this process [33]. In this paper, we demonstrated that the LlQRT2 transcript level was positively controlled by $\mathrm{GA}_{3}$. The level of LIQRT2 mRNA increased, especially in the second LAD phase, which suggests GA-dependent modulation of the septum/stomium interruption in the studied plant. Data on the impact of GAs on $P G$ expression in different plant species are lacking. In $A$. thaliana anthers, the application of another phytohormone, JA, causes an increase in QRT2, ADPG1 (ARABIDOPSIS DEHISCENCE ZONE POLYGALACTURONASE1) and ADPG2 expression by approximately 10 -fold [19]. In wheat (T. aestivum), cisacting elements in the promotor sequences of $T a P G$ genes were predicted [68]. Importantly, they included ciselements regulated by phytohormones and transcription factors such as abscisic acid (ABA; ABRE), GAs (P-Box), auxin (TGA-element), methyl jasmonate (MeJA; CGTCA, TGACG), and MYB binding sites involved in drought inducibility (MBS). Similar research was carried out in Brassica oleracea, and hormone-response cis-elements were found for MeJA (CGTCA), auxin (TGA-box and AuxRR-core), GA (GARE, P-box, TATC-box), salicylic acid (TCA-element), and ABA (ABRE) [69].

The separated septum cells of yellow lupine progressively degenerate. The septum degenerates first to create a bilocular anther, and the stomial cells then degenerate to break the anther wall. In other plant species, these processes occurring in specified cells are aided by PCD [1, 8]. Ge et al. (2005) showed that the PCD inhibitory factor gene PCS1, encoding 
aspartic protease, promotes cell survival during embryogenesis and gametogenesis. PCS1 is not continuously expressed in tissues but is involved in various specific developmental processes. A characteristic pattern of PCS1 expression has been demonstrated in developing flowers, young siliques, and anthers. Further analyses revealed the presence of PCS1 mRNA in pollen and the anther wall, with the exception of the tapetum layer, where no information has been provided [21]. The overexpression of PCS1 in A. thaliana anthers results in the inhibition of the dehiscence process; therefore, we focused on establishing the expression of this gene in yellow lupine tissues. Our results show that the level of LlPCS1 fluctuates at different stages of LAD. It is extremely interesting that LlPCS1 transcriptional activity is controlled by $\mathrm{GA}_{3}$. As expected, hormone molecules reduced the amount of LlPCS1 mRNA, especially in the first and fourth LAD phases, which indirectly proves the GAdependence of septum/stomium cell degeneration in yellow lupine. In summary, the results suggest that PCD-related processes occur during the development of yellow lupine anthers, but the mechanisms of these strictly controlled processes require further research. In various plant species, two main types of stomium rupture were recognized, basic mechanical breakage and active PCD-related degeneration [8, 9, 70]. Developmental PCD is controlled by phytohormones. Sanders et al. (2000) showed that the mutant associated with the JA biosynthetic pathway opr3 is characterized by a delay in the degeneration of stomium cells, which disturbs anther dehiscence [9]. Thus, there is strong indirect proof of the involvement of these phytohormones in $\mathrm{PCD}$ and in the process of anther opening.

\section{Gibberellins are modulators of anther dehiscence in yellow lupine}

The multifunctionality of GAs in the early stages of anther development, mainly tapetum and pollen, is well documented through analyses of $A$. thaliana and rice mutants. However, the late stages of anther development related to their final split are not well understood due to the earlier GA-deficiency mutant disturbances that prevent further anther development [71]. In yellow lupine, expression profile studies have shown that fluctuating transcript levels of LIDELLA1, which encodes the main repressor of GA signalling, promote proper flower and pod development. The LIDELLA1 mRNA level is lowest when the anthers are opened and then increases during the fertilization and early pod developmental stages [34]. This encouraged us to investigate the localization of $\mathrm{GA}_{3}$ in selected stages of late anther development in yellow lupine. Our results obtained in this work closely correlate with the expression pattern of the LIDELLA1 gene, in the opposite manner. The highest accumulation of $\mathrm{GA}_{3}$ occurs in the first LAD phase, which is before anther opening. The $\mathrm{GA}_{3}$ signal was observed mainly in degenerating septum cells, near the vascular bundle, in the middle layer, endothecium, and less frequently in the epidermis. In the second LAD phase, where the stomium cells were disrupted and the pollen chambers were opened, the fluorescence signal was almost unnoticeable, and no signal was detected in the third and fourth LAD stages. In the case of rice, $\mathrm{GA}_{4}$ accumulation was found to be highest in anthers just before anthesis. The level of active hormone molecules decreased, and was completely undetectable in the young seeds a week after anthesis [72]. These results suggest that GAs play an important role in specific organs (place) at a specific stage of the life cycle (time) and that they may comprise the strict regulation of reproductive growth and development in different species.

The application of the GA biosynthesis inhibitor PAC in petunia (Petunia hybrida) causes male sterility resulting from the inhibition of anther development in the postmeiotic phase. Detailed analyses showed that the connective cells and tapetum were degenerated, but pollen grains were still present. In petunia transgenic plants overexpressing the GA signalling repressor AtSPY, the anther phenotype was comparable to that observed after PAC treatment [73]. In some plants, inhibition of GA biosynthesis blocks anther development after meiosis (petunia, A. thaliana, maize), but in others, blockage occurs before meiosis, i.e., tomato [71]. The key and final gene involved in GA biosynthesis is GA3ox. We showed that the transcriptional activity of LlGA3ox strictly correlates with $\mathrm{GA}_{3}$ localization in yellow lupine during late anther development. The most LlGA3ox transcripts were found in the first and second LAD stages, and the level decreased significantly in the third and fourth stages. Moreover, after the application of PAC, the amount of LlGA3ox mRNA decreased by more than half, mainly in the first three selected phases. This suggests that this particular LlGA3ox gene may be involved in the synthesis of bioactive phytohormone molecules that play an essential role in the studied process in yellow lupine. In addition, we demonstrated that the expression of LlGA2ox1, encoding the enzyme responsible for GA inactivation, shows the opposite expression to that of LlGA3ox. The role of genes involved in GA biosynthesis and inactivation during stamen development is well understood in many plant species. The transcriptional activity of AtGA3ox1AtGA3ox4 genes was observed in stamens, and AtGA3ox 1 was also expressed in some other floral organs. Both the stamen filaments and anthers require GAs for proper development, and de novo synthesis of 
this hormone was confirmed at both sites. The transcript of $A t G A 3 o x 1$ was found in the filament, and $A t G A 3 o \times 2-$ AtGA3ox4 genes were expressed in anthers and pollen [26]. GAs, mainly $\mathrm{GA}_{4}$, need to be transported from stamens or flower receptacles to other floral organs to ensure proper development. Despite the lack of expression of all $A t G A 3 o x$ genes in the petals, the mutant deficient in AtGA3ox1 and AtGA3ox3 showed serious defects in petal development [26]. In yellow lupine anthers, this could be explained by the observation of a fluorescent signal indicating the presence of $\mathrm{GA}_{3}$ in the cells of the conductive bundle. Therefore, there is a high probability that the active phytohormone molecules are transported from the anther to the filament or vice versa. An analogous situation was observed in petunia, where GA application restored the normal corolla pattern, previously disturbed by removal of the stamens [74]. Similarly, anther expression patterns of GA3ox genes were also observed in tobacco and rice [75, 76]. In summary, stamens are a key source of GAs in flowers in different plant species. In addition to their local influence on stamen development, their transport to adjacent tissues of flower organs is equally important [26].

The GAMYB transcription factor mediates GA signal transduction in the aleurone tissues of cereals. Furthermore, it is necessary to ensure the fertility of anthers in many species. Similar to GA biosynthesis mutants, gamyb demonstrates the impact of GA signalling on the early stages of anther development. A mutation in GAMYB in rice and the double mutation of MYB33 and MYB65 in A. thaliana interrupt PCD, causing abnormal enlargement of tapetum cells, which disorders pollen development [28]. However, there are few data concerning the role of $G A M Y B$ in late anther development. In this paper, we examined yellow lupine $L l G A M Y B$ expression in selected phases of LAD and after $\mathrm{GA}_{3}$ and PAC application. The $L l G A M Y B$ transcript level was almost identical with or without $\mathrm{GA}_{3}$ treatment, but PAC application resulted in increased $L l G A M Y b$ mRNA, especially in the first and second LAD phases. This suggests that the $\mathrm{GA}_{3}$ dependent pathway may control LlGAMYB expression but not in a direct manner. It can be assumed only that the presence of $\mathrm{GA}_{3}$ blocks the transcription factor, which positively regulates the expression of $L l G A M Y B$, but the exact mechanism requires further research. The results obtained in other plant species show that $H \nu G A$ $M Y B$ in barley and GAMYB-like genes in A. thaliana are expressed in flowers and are positively regulated by GAs $[29,77]$. It follows that the role of GAMYB in GAregulated gene expression during anther development differs between species. Moreover, the function of $G A M Y B$ also varies within a species between developmental processes, e.g., seed germination and vegetative and reproductive development [78]. Interestingly, the results of studies carried out on barley, indicate that transgenic plants strongly overexpressing $H \nu G A M Y B$ in anthers are completely male sterile. This phenotype was characterized by an intact septum [29]. Taking into account our results obtained in yellow lupine, where we observed the highest level of $\mathrm{GA}_{3}$ in progressively degenerating septum cells, it can be concluded that the presence of $\mathrm{GA}_{3}$ at this stage of anther development strictly regulates the level of target gene transcripts (establishing a certain balance), which results in a specific and correct physiological response. Pollen of HVGAMYB-overexpressing barley anthers has a specific phenotype [29]. The development of slightly smaller and irregular pollen is normal, and some pollen has been shown to be viable. The observed male sterility is mainly due to disrupted anther wall tissues, which do not break [29]. Similar to barley, a phenotype of small, indehiscent anthers was observed in wheat after $\mathrm{GA}_{3}$ treatment $[79,80]$. The occurrence of male sterility following the application of $\mathrm{GA}_{3}$ in wheat, in conjunction with the observations that (1) $H \nu G A M Y B$ overexpression causes anther indehiscence and that (2) LlGAMYB expression is relatively constant after $\mathrm{GA}_{3}$ application but increases after PAC treatment, suggest that GAMYB plays a specific role during anther development in a species-specific manner. This requires further research and more clarification.

Many reports have suggested that the expression of GAMYB-like genes in A. thaliana is regulated posttranscriptionally by a microRNA (miRNA) named miR159 [30, 81]. miR159 is a small (21 nt), noncoding RNA sequence derived from double-stranded RNA (dsRNA). At-miR159 affects the transcriptional activity of its target genes, including AtMYB33, AtMYB65 and AtMYB101 [30]. In rice, Tsuji et al. (2006) revealed that miR159 regulates the transcript level of GAMYB only in flowers, and this situation was not observed in aleurone cells. It follows that the regulation of $G A M Y B$ expression and its function is organ specific [78]. Due to this fact, and also to find a factor that may determine the level of $L l G A M Y B$ transcripts during anther development in yellow lupine, we examined LIMIR159 expression after $\mathrm{GA}_{3}$ and PAC application. Interestingly, $\mathrm{GA}_{3}$ treatment increased the LlMIR159 transcript content, and PAC application significantly decreased it. The observed expression profile of LIMIR159 partially explains the transcriptional activity changes observed for $L l G A M Y B$ after different compound applications. The results also indicate that the expression of the LIMIR159 gene is regulated via the GA signalling pathway. A similar situation occurs in A. thaliana, where GAs regulate miR159 levels [30]. In the flowers of GA biosynthesis mutant $g a 1-3$, there was significantly less miR159 than in the WT flowers. Application of GAs to the flowers of the mutant increased the level of miR159 to a level comparable to that of GA-treated WT plants, and this level 
was higher than that of untreated WT plants. Due to the reduced level of AtMYB33, plants overexpressing miR159 showed male sterility and delayed flowering [30]. As demonstrated by experiments with the GUS reporter gene, the localization of AtMYB33:GUS encoded by a gene with the complete miR159 target sequence was only in young anthers whereas AtMYB33:GUS was expressed in various flower organs when the gene had damage to the miR159 target sequence [81].

By analysing the natural conditions without the application of any compounds, it can be concluded that LlGAMYB expression is high in the first two stages of LAD and then decreases. This negatively correlates with the transcriptional activity of LIMIR159, which was significantly increased in the second phase of LAD. This is probably the cause of the reduced mRNA content of $L l G A M Y B$ in the third and fourth LAD stages. The results obtained suggest that $L I G A M Y B$ is coexpressed with LIMIR159 in yellow lupine anthers. It also follows that the $L l G A M Y B$ gene is regulated by miR159 in the anther of yellow lupine.

\section{In silico analyses}

In this paper, nine full-length cDNA sequences of genes related to the anther dehiscence process in yellow lupine were identified. The implemented methodology was successful for the identification and characterization of the conserved domains, motifs and specific amino acids of all predicted proteins. This approach made it possible to predict and assign them specific functions. The identified genes encode functional proteins closely related to the Fabaceae family. Moreover, multiple protein alignments provided information about the conservation of sequences in many plant species. It also allowed for the specification of fragments of protein sequences that are the crucial for the function they perform. The construction of phylogenetic trees allowed for making conclusions about the origin of the predicted yellow lupine proteins.

Based on the available bioinformatics and experimental results, miR159 mediates the regulation of the family of GAMYB and GAMYB-like genes in various plant species, including $A$. thaliana [81], rice [78] and strawberry [82]. These $G A M Y B$ genes from different plant species and LlGAMYB identified in this work from yellow lupine contain a conserved miR159 binding site, which is highly complementary to the corresponding miR159 sequence and is located in the conserved region between Box 1 and Box 2 of GAMYB genes [78, 81]. This indicates that the miR159-GAMYB pathway is conserved across species.

\section{Conclusions}

This paper advances knowledge of the hormonal and molecular regulation of late anther development in the cleistogamous plant yellow lupine (Lupinus luteus L); such knowledge is an important aspect of controlling fertility in this valuable legume crop species. To date, there are no data showing the effect of GAs on the transcriptional activity of genes associated with deposition of lignocellulosic secondary thickening in the endothecium, enzymatic breakdown of cell walls at the septum/stomium and cell degeneration via PCD-related processes. Therefore, we showed that the temporal expression of the identified genes, as markers of developmental changes during yellow lupine anther dehiscence, is regulated in a GA-dependent pathway. Additionally, tissue and cellular localization suggests that $\mathrm{GA}_{3}$ is a modulator of this process, especially in the time prior to anther opening. The appropriate $\mathrm{GA}_{3}$ level, which correlates with GA metabolism, at the right time and place controls yellow lupine anther opening, likely through the influence on $L l G A M Y B$ expression via the Ll-MIR159dependent pathway. Therefore, yellow lupine anther dehiscence is highly regulated, enabling the timing of pollen release to be tightly controlled to maximize the chances of fertilization.

\section{Methods}

Plant material, growing conditions and compound treatments

The plant material was yellow lupine (Lupinus luteus L.) cv. Taper. Seeds obtained from the Plant Breeding Station Wiatrowo (Poland) were prepared and sown as described in previous research [34] in the experimental field in Pędzewo, Kuyavian-Pomeranian Voivodeship in north-central Poland $\left(53^{\circ} 05^{\prime} 02^{\prime \prime} \mathrm{N} 18^{\circ} 21^{\prime} 28^{\prime \prime} \mathrm{E}\right)$. The plants were cultivated in soil of the 5 th class (poor arable soil; Polish bonitation classification of soils) as recommended by the manufacturer [83]. The anthers from particular stages of late development (1-4 LAD, Fig. 1A) were dissected from flowers with a sharp scalpel. For each phase of LAD, no less than 80 plants were used, and part of the collected material was previously treated with gibberellic acid $\left(\mathrm{GA}_{3}\right.$, $100 \mu \mathrm{M}$ ) or GA biosynthesis inhibitor paclobutrazol (PAC, $100 \mu \mathrm{M})$ in a $0.05 \%$ Tween 20 solution using a sprayer. The anthers in the corresponding developmental phases were used as the control group, and were treated with $0.05 \%$ Tween 20 solution only. According to the planned experiment, $3 \mathrm{~h}$ after application, the anthers were harvested and processed fresh or frozen in liquid nitrogen (and stored at $-80^{\circ} \mathrm{C}$ until use).

\section{Identification of CDNAs}

The cDNA sequences of individual genes were identified differently. Molecular cloning of LlGA2oxl cDNA was performed as described in prior research [33]. The identification of $L l G A M Y B$ cDNA was as follows: $1 \mathrm{~g}$ of yellowish-green flowers was pounded in liquid nitrogen using a mortar and pestle. Then total RNA was isolated 
by the column method according to the manufacturer's instructions (NucleoSpin ${ }^{\circ}$ RNA kit, Macherey-Nagel, Düren, Germany), and reverse transcription was performed using $1 \mu \mathrm{g}$ of RNA, oligo $(\mathrm{dT})_{18}$ primers, and the Transcriptor High Fidelity cDNA Synthesis Kit (Roche, Mannheim, Germany). Touchdown PCR was performed using 1.25 U Perpetual Taq DNA Polymerase ${ }^{\text {HOT START }}$ (EURx, Gdańsk, Poland), $2 \mu \mathrm{l}$ of first-strand cDNA, $1 \mathrm{x}$ buffer B, $0.2 \mathrm{mM}$ dNTP mix, $3.0 \mathrm{mM} \mathrm{Mg}{ }^{2+}, 1 \mu \mathrm{M}$ degenerated primers (Tab. S1) and deionized water (up to a final volume of $50 \mu \mathrm{l}$ ) in a T3 thermocycler (Biometra, Göttingen, Germany). An amplified cDNA fragment (704 bp, Fig. S13A) was isolated and purified from an agarose gel (GeneMATRIX Agarose Out DNA Purification Kit, EURx). At the insertion site, the PCR product was ligated into the pCRII-TOPO vector (Fig. S13B, TOPO TA Cloning Kit, Invitrogen, Carlsbad, USA) and transferred into One Shot Mach1-T1 E. coli in the form of a plasmid. The bacterial cells were plated on Petri dishes containing S-Gal/LB agar blend (Sigma-Aldrich, St. Louis, MO, USA) with ampicillin $(50 \mu \mathrm{g} / \mathrm{ml})$ (Fig. S13C). Unlike dark blue bacterial colonies, white colonies were selected and cultured in liquid LB medium with ampicillin $(50 \mu \mathrm{g} / \mathrm{ml})$ for $12 \mathrm{~h}$. Finally, plasmid DNA was isolated in accordance with the manufacturer's guidelines (GeneMATRIX Plasmid Miniprep DNA purification kit, EURx), digested with the restriction enzyme EcoRI (Fermentas) (Fig. S13D), and sequenced by Genomed (Warsaw, Poland). The 485 bp (Fig. S14A) and 768 bp (Fig. S14B) fragments were obtained from 3' RACE-PCR (FirstChoice RLM-RACE Kit, SuperTaq-Plus Polymerase, Ambion, Austin, USA) using two different pairs of primers (Tab. S1). The amplicons were isolated, purified, cloned, digested (Fig. S14C/D) and sequenced as described above. Due to difficulties arising from the experimental identification of the $5^{\prime}$ end of $L l G A M Y B$ cDNA, it was obtained based on sequences derived from a later RNA-Seq experiment deposited at the NCBI in the Sequence Read Archive (SRA) database under accession number PRJNA285604 (BioProject) [84] and experiment accession number SRX1069734. All fragments had overlapping nucleotide sequences which allowed for us to obtain the complete $L l G A$ $M Y B$ sequence. The de novo assembled transcriptome of yellow lupine from RNA-seq experiments was also used to identify $L l C A D$, all LlIRXs, LIQRT2, LlPCS1, LlGA3ox1 and the precursor of Ll-miR159 (Ll-MIR159).

\section{Expression analysis}

Quantitative real-time PCR (qPCR) was used to establish the expression pattern of all identified genes. Eighty milligrams of frozen anthers (at a specific stage of development with or without $\mathrm{GA}_{3} / \mathrm{PAC}$ treatment) were homogenized in a sterile mortar with a pestle. According to the manufacturer's instructions, total RNA was isolated using an Isolate II RNA Plant Kit (Bioline, London, UK), and reverse transcription with matrix $(2 \mu \mathrm{g})$, oligo $(\mathrm{dT})_{18}$ (Roche) and the Transcriptor High Fidelity cDNA Synthesis Kit was performed. qPCRs were carried out using a LightCycler 2.0 Carousel-Based System (Roche) in $20 \mu \mathrm{l}$ capillaries containing a mix of $0.1 \mu \mathrm{g}$ of cDNA, $0.2 \mu \mathrm{M}$ gene-specific primers (Tab. S1), $0.05 \mu \mathrm{M}$ Universal Probe Library (UPL) hydrolysis probes (Roche) (Tab. S1), $1 \times$ LightCycler TaqMan Master Mix (LightCycler TaqMan Master Kit, Roche) and $\mathrm{H}_{2} \mathrm{O}$. The following program was used: $600 \mathrm{~s}$ at $96^{\circ} \mathrm{C} ; 45$ cycles of $10 \mathrm{~s}$ at $96^{\circ} \mathrm{C}, 20 \mathrm{~s}$ at a specific annealing temperature (Tab. S1), $1 \mathrm{~s}$ at $72{ }^{\circ} \mathrm{C}$; and $30 \mathrm{~s}$ at $40^{\circ} \mathrm{C}$. Negative no template controls (NTCs) were included. As an endogenous control the actin gene (LlACT, GenBank accession number KP257588) was selected [32-34]. Absolute quantification was designed from the serial dilutions of cDNAs generating standard curves, and the relative gene expression (the data of the studied gene relative to the internal control gene and calibrator) was determined using the 2 (-DeltaDeltaC(T)) method [85].

\section{Histological studies}

A fixer containing $4 \%$ paraformaldehyde $(\mathrm{w} / \mathrm{v}), 0.2 \%$ glutaraldehyde (v/v) and 3\% N-ethyl- $\mathrm{N}^{\prime}$-(3-dimethylaminopropyl) carbodiimide hydrochloride (EDAC) (w/v) (Sigma-Aldrich) in $1 \times$ phosphate-buffered saline buffer (1× PBS, pH 7.2) was prepared and applied to appropriate anther tissue small fragments for $12 \mathrm{~h}$ at $4{ }^{\circ} \mathrm{C}$. Triplicate samples were washed of fixative with $1 \times \mathrm{PBS}(\mathrm{pH}$ 7.2) for $10 \mathrm{~min}$. Dehydration was achieved by placing the material in increasing ethanol concentrations $(30,50,70$, $90,100 \%)(\mathrm{v} / \mathrm{v})$. Then, supersaturation and embedding in BMM resin (butyl methacrylate, methyl methacrylate, $0.5 \%(\mathrm{w} / \mathrm{v})$ benzoin ethyl ether, $10 \mathrm{mM}$ dithiothreitol) (Fluka, Buchs, Switzerland) were performed at $-20^{\circ} \mathrm{C}$ under UV light. Using an ultramicrotome (ReichertJung, Germany) semithin sections were obtained, which were placed on slides, stained with $0.05 \%$ toluidine blue (Sigma-Aldrich), and observed under an LM Zeiss Axioplan (Carl Zeiss, Oberkochen, Germany) microscope with a ProGres C3 digital camera.

\section{$\mathrm{GA}_{3}$ immunolocalization}

The anther tissue fragments were fixed, washed, dehydrated, supersaturated, embedded, polymerized and cut in the same way as described for the histological studies. Semithin sections were placed on slides with Biobond (BBInternational, Cardiff, UK), and after washing, the resin was blocked in BlockAid TM Blocking Solution (Thermo Fisher Scientific, Waltham, MA, USA) according to the manufacturer's instructions. Then, sections were 
incubated with polyclonal primary antibody anti-GA 3 (Abbexa, Cambridge, UK) diluted 1:50 in 1\% bovine serum albumin (BSA) in 1x PBS (pH 7.2) and placed in a wet container at $4{ }^{\circ} \mathrm{C}$ for $12 \mathrm{~h}$. Next, the primary antibodies were removed by washing 3 times in $1 \mathrm{x}$ PBS ( $\mathrm{pH} 7.2$ ), and secondary antibody (MFP488 goat anti-rabbit IgG, MoBiTec, Goettingen, Germany) diluted 1:250 in PBS buffer was applied for $2 \mathrm{~h}$ at $37^{\circ} \mathrm{C}$. A negative control was performed by omitting incubation with the primary antibody (Fig. S15). The final steps were incubation with DAPI (1: 2500) for $10 \mathrm{~min}$ and washing with distilled water. The samples were observed under a Leica DMI4000B inverted microscope using BP365, FT395, and LP397 filters.

\section{Is silico analyses}

The integrated FastPCR v.6.5.99 [86] tool was used to design the degenerate and RACE-PCR primers. The Universal Probe Library Assay Design Center [87] was used to design the qPCR-specific primers and short hydrolysis UPL probes substituted with locked nucleic acids. The identified cDNA sequences of all genes and predicted proteins were analysed using the Basic Local Alignment Search Tool (BLAST) [88] and the bioinformatics resource portal of the Swiss Institute of Bioinformatics [Expasy [89], including the Translate tool [90], which allows for the translation of nucleotide sequences to protein sequences, and the ProtParam tool [91], which allows the calculation of molecular weights and isoelectric points. Alignments and phylogenetic reconstructions were performed using the Python Environment for Tree Exploration3 (ETE3) v3.1.1 program as implemented in GenomeNet [92]. Maximum likelihood phylogenetic trees were inferred using PhyML v20160115 ran with model and parameters: --pinv e -o tlr -f $m$--bootstrap - 2 --nclasses 4 --alpha e. Branch supports are the $\mathrm{Chi}^{2}$-based parametric values return by the approximate likelihood ratio test. Multiple alignments of different amino acid sequences found in BlastP and showing close association with yellow lupine sequences were carried out using the ClustalW [93] program with the default settings. The presence of functional domains was checked via the NCBI Conserved Domain Database (CDD) [94], a protein annotation resource consisting of a collection of well-annotated multiple sequence alignment models for ancient domains and full-length proteins. These are available as position-specific score matrices (PSSMs) for the rapid identification of conserved domains in protein sequences via RPS-BLAST. The CDD content includes NCBIcurated domains as well as domain models imported from a number of external source databases (Pfam, SMART, COG, PRK, TIGRFAMs). The cytoplasmic and transmembrane domains were also predicted using TMHMM v. 2.0 [95] and the Protein Homology/analogY
Recognition Engine V 2.0 (Phyre $^{2}$ ) [96] web portal for protein modelling, prediction and analysis. Comparisons of proteins derived from different plant species were made using the DiAlign program (Genomatix) [97] with default parameters. The tertiary structures of the proteins derived from yellow lupine were constructed using the Robetta service for protein structure prediction [98], which uses the PDB100 template database and a coevolution-based model database (MDB). The results were visualized using UCSF ChimeraX [99], which is a next-generation molecular visualization program from the Resource for Biocomputing, Visualization, and Informatics (RBVI). The Ll-pre-miR159 sequence was analysed using the microRNA database (miRBase), ETE3 and BLAST. RNA structure software [100] was used to compute the secondary structure of Ll-pre-miR159.

\section{Statistical analysis}

All presented data are the results of three separate samples (biological replications) with two repetitions of each (technical replications) and are presented as the mean \pm standard error (SE). Statistical analysis was performed using one-way ANOVA followed by post hoc Tukey's HSD test, with differences accepted at $p<0.05$. All analyses were performed using $\mathrm{R}$ version 3.5.3.

\section{Abbreviations}

ACT: actin; ADPG1/2: Arabidopsis dehiscence zone polygalacturonase 1/2; AHP4: Arabidopsis histidine-containing phosphotransfer 4; C: Connective;

CAD: Cinnamyl alcohol dehydrogenase; CesA8: Cellulose synthase A catalytic subunit 8 [UDP-forming]; CKs: Cytokinins; COBL4: COBRA-like 4; E: Epidermis; En: Endothecium; GA: Gibberellic acid; GA3ox: Gibberellin 3-oxidase; GA2ox: Gibberellin 2-oxidases; GAs: Gibberellins;

GAUT12: Galacturonosyltransferase 12; GPI: Glycosylphosphatidylinositol; GT8: Glycosyl transferase family 8; IRX1/6/8: Irregular xylem 1/6/8; LAD: Late anther development; PAC: Paclobutrazol; PCD: Programmed cell death; PCS1: Promotion of cell survival 1; P: Pollen grain; PGs: Polygalacturonases; QRT2: Quartet 2; Se: Septum; St: Stomium; StR: Stomium region; T: Tapetum; VB: Vascular bundle

\section{Supplementary Information}

The online version contains supplementary material available at https://doi. org/10.1186/s12870-021-03085-4.

\footnotetext{
Additional file 1: Fig. S1a. LICAD cDNA (GenBank accession number MW240676, 2250 bp) identified in yellow lupine (Lupinus luteus L.) and its deduced amino acid sequence [357 aa (ExPASy, translate tool), m.w. = $38.936 \mathrm{kD}$ and $\mathrm{pl}=6.01$ (ExPASy, ProtParam)]. The nucleotides are marked with lowercase letters, and amino acids are marked with capital letters. The START and STOP codons (yellow background) are indicated. $5^{\prime}$ and $3^{\prime}$ UTR regions are marked with small italic letters before the ATG and after the TGA codons, respectively. The ORF is shown in pink. Fig. S1b. Maximum likelihood phylogenetic tree of 15 cinnamyl alcohol dehydrogenase (CAD) proteins (BlastP) with the highest degree of similarity to LICAD. Alignment and phylogenetic reconstructions were performed using the Environment for Tree Exploration3 (ETE3) v3.1.1 program as implemented in GenomeNet. The ML tree was inferred using PhyML v20160115. Branch supports are the Chi ${ }^{2}$-based parametric values returned by the approximate likelihood ratio test. Fig. S1c. Multiple alignment (ClustalW) of 15 cinnamyl alcohol dehydrogenase (CAD) amino acid sequences (BlastP) that are closely related to LICAD. The alcohol
} 
dehydrogenease GroES-like domain is shaded medium grey, and the zinc-binding dehydrogenase domain is shaded dark grey. The $\mathrm{Zn}-1$ and Zn-2 binding motifs are shaded with violet. Three green amino acids, $C$, $\mathrm{H}$, and $\mathrm{C}$, are marked with green dots. Yellow letters define four cysteine (C) residues. Conserved glycine $(\mathrm{G})$ residues $(\mathrm{G} \times \mathrm{GG} \times \mathrm{G})$ are indicated with red letters. They represent NADPH cosubstrate-binding motif, which is highlighted in green. The conserved residues ( $, Q, L, M, W, V, P, L, F, I)$ are highlighted in blue. The serine (S) 212 is labelled with a red background. Most of the alignment information was identified according to the results described by [38-40]. Lang - Lupinus angustifolius

(XP_019452456); Lalb - Lupinus albus (KAE9589372); Ap - Abrus precatorius (XP_027363622); Vu - Vigna unguiculata (XP_027937462); Gs - Glycine soja (XP_028185029); Gm - Glycine max (XP_003555961); Ss - Spatholobus suberectus (TKY50969); Ck - Caragana korshinskii (AEV93476); Ah - Arachis hypogaea (XP_025620500); Ad - Arachis duranensis (XP_015941997); Ai Arachis ipaensis (XP_016175492); In - Ipomoea nil (XP_019169368); Mt Medicago truncatula (XP_013470061); Cc - Cajanus cajan (XP_020210039); At - Arabidopsis thaliana (AAK44076). Fig. S1d. Comparison of CAD proteins derived from different plant species (BlastP) using the DiAlign program (Genomatix). For each pairwise alignment, the similarity (relative to the maximum similarity) and the number of identical amino acids (in \% of shorter sequence) are given. Maximum values are underlined. The similarity value of 1.000 denotes only the two most similar sequences; it does not necessarily mean that these sequences are identical. Lang - Lupinus angustifolius (XP 019452456); Lalb - Lupinus albus (KAE9589372); Ap Abrus precatorius (XP_027363622); Vu - Vigna unguiculata (XP_027937462); Gs - Glycine soja (XP_028185029); Gm - Glycine max (XP_003555961); Ss Spatholobus suberectus (TKY50969); CK - Caragana korshinskii (AEV93476); Ah - Arachis hypogaea (XP_025620500); Ad - Arachis duranensis (XP_015941997); Ai - Arachis ipaensis (XP_016175492); In - Ipomoea nil (XP_019169368); Mt - Medicago truncatula (XP_013470061); Cc - Cajanus cajan (XP_020210039); At - Arabidopsis thaliana (AAK44076). Fig. S2a. LICesA8 (alternative name LIIRX1) CDNA (GenBank accession number MW240677, 3788 bp) identified in yellow lupine (Lupinus luteus L.) and its deduced amino acid sequence [975 aa (ExPASy, translate tool), m.w. = $110.227 \mathrm{kD}$ and $\mathrm{pl}=5.97$ (ExPASy, ProtParam)]. The nucleotides are marked with lowercase letters, and amino acids with capital letters. The START and STOP codons (yellow background) are indicated. 5' and 3' UTR regions are marked with small italic letters before the ATG and after the TAA codons, respectively. The ORF is shown in pink. Fig. S2b. Maximum likelihood phylogenetic tree of 12 CesA8 proteins (alternative name IRX1, BlastP) with the highest degree of similarity to LICesA8/LIIRX1. Alignment and phylogenetic reconstructions were performed using the ETE3 v3.1.1 program as implemented in GenomeNet. The ML tree was inferred using PhyML v20160115. Branch supports are the Chi'-based parametric values returned by the approximate likelihood ratio test. Fig. S2c. Multiple alignment (ClustalW) of 12 CesA8/IRX1 amino acid sequences (BlastP) that are closely related to LICesA8/LIIRX8. Proteins contain N-terminus; globular/ soluble central domain $(C D)$ and the $\mathrm{C}$-terminus. Short $\mathrm{N}$-terminus $(\mathrm{N})$ is prior to the Zinc-binding domain (Zn) (medium gray). This $Z n$ domain contains strictly conserved $\mathrm{CxxC}$ motif (green box and red letters) beginning amino acids: $C x x C x_{12} F x A C x x C x x P x C x x C x E x x x x x D x x x C x x C$, where $x$ is any amino acid. Within the $\mathrm{N}$-terminus is also a region rich in acidic amino acids designated as a hypervariable region (VR1, red box). Following the $\mathrm{N}$-terminal domains are two transmembrane domains ( $\mathrm{TMH} 1 / 2$, yellow boxes). The CD contains variable region 2 (VR2, dark grey) composed mostly of the Class Specific Region (CSR) which on either side is flanked by conserved regions: CR1 [with the Plant Conserved Region (P$(\mathrm{CR})$ in the middle highlighted in light grey)] and CR2. The four recognized catalytic motifs are marked as blue boxes, with the D, DxD, and D residues in bold red and the QxxRW residues in bold orange. The numerous basic residues of $\operatorname{Arg}(R)$ and Lys $(K)$ (green leterrs) and acidic residues of Asp (D) and Glu (E) (red letters) of the VR2/CSR, as well as the conserved Cys (C, yellow letters) are conserved across species in an isoform-specific manner [43]. The C-terminus contains six transmembrane domains (THM 3-8) and remaining protein after the last TM helix marked as C. Fig. S2d. Comparison of CesA8 proteins derived from different plant species using the DiAlign program (Genomatix). For each pairwise alignment, the similarity (relative to the maximum similarity) and the number of identical amino acids (in \% of shorter sequence) are given. Maximum values are underlined. The similarity value of 1.000 denotes only the two most similar sequences; it does not necessarily mean that these sequences are identical. Lang - Lupinus angustifolius (XP_019439632); Gm -Glycine max (XP_003526279); Mt - Medicago truncatula (XP_013462596); Va - Vigna angularis (XP_017421931); Cc - Cajanus cajan (XP_020211247); Ap - Abrus precatorius (XP_027339063); Ad - Arachis duranensis (XP_015943020); Ah - Arachis hypogaea (XP_025621820); Gs - Glycine soja (KHN48007); Jc - Jatropha curcas (XP_012091811); Lalb - Lupinus albus (KAE9602950); At - Arabidopsis thaliana (NP_567564). Fig. S3a. L/COBL4 (alternative name LIIRX8) cDNA (GenBank accession number MW240678, $2370 \mathrm{bp}$ ) identified in yellow lupine (Lupinus luteus L.) and its deduced amino acid sequence [431 aa (ExPASy, translate tool), m.w. $=48.489 \mathrm{kD}$ and $\mathrm{pl}=8.85$ (ExPASy, ProtParam)]. The nucleotides are marked with lowercase letters, and amino acids with capital letters. The START and STOP codons (yellow background) are indicated. 5' and $3^{\prime}$ UTR regions are marked with small italic letters before the ATG and after the TGA codons, respectively. The ORF is shown in pink. Fig. S3b. Maximum likelihood phylogenetic tree of 14 COBRA-like 4 (COBL4) / irregular xylem 6 (IRX6) proteins (BlastP) with the highest degree of similarity to LICOBL4/LIIRX6. Alignment and phylogenetic reconstructions were performed using the ETE3 v3.1.1 program as implemented in GenomeNet. The ML tree was inferred using PhyML v20160115. Branch supports are the Chi ${ }^{2}$-based parametric values returned by the approximate likelihood ratio test. Lang Lupinus angustifolius (XP_019452983); CC - Cajanus cajan (XP_020240142); Ap - Abrus precatorius (XP_027353541); Ss - Spatholobus suberectus (TKY65050); Mp - Mucuna pruriens (RDX75309); Ad - Arachis duranensis (XP_015946272); Ai - Arachis ipaensis (XP_016182359); Vr - Vigna radiata (XP_014522888); Gs - Glycine soja (XP_028215469); Ah - Arachis hypogaea (XP_025624246); Mt - Medicago truncatula (XP_013451095); Gm - Glycine max (XP_003554956); Pa - Prosopis alba (XP_028764818); At - Arabidopsis thaliana (NP_197067). Fig. S3c. Multiple alignment (ClustalW) of 14 COBL4/IRX6 amino acid sequences (BlastP) that are closely related to LICOBL4/LIIRX6. Conserved domain characteristic to COBRA superfamily is marked in green background. The $\mathrm{N}$-terminal predicted signal peptide is marked yellow, with cleavage site marked by black arrow. The underlined sequences with red letters show the putative cellulose-binding sites. The light blue sequences denote the Cys-rich (CCVS) motif characteristic for all COBL proteins, while CCVS amino acids are marked in dark blue background and white letters. Two conserved consensus N-glycosylation sites are indicated by a white letters on violet backgrounds. Locus corresponds to the predicted cleavage $\omega$ sites in the $C$ terminus is indicated in middium grey background and black arrow. Fig. S3d. Comparison of COBL4/ IRX6 proteins (BlastP) derived from different plant species using the DiAlign program (Genomatix). For each pairwise alignment, the similarity (relative to the maximum similarity) and the number of identical amino acids (in \% of shorter sequence) are given. Maximum values are underlined. The similarity value of 1.000 denotes only the two most similar sequences; it does not necessarily mean that these sequences are identical. Lang - Lupinus angustifolius (XP_019452983); CC - Cajanus cajan (XP_020240142); Ap - Abrus precatorius (XP_027353541); Ss - Spatholobus suberectus (TKY65050); Mp - Mucuna pruriens (RDX75309); Ad - Arachis duranensis (XP_015946272); Ai - Arachis ipaensis (XP_016182359); Vr - Vigna radiata (XP_014522888); Gs - Glycine soja (XP_028215469); Ah - Arachis hypogaea (XP_025624246); Mt - Medicago truncatula (XP_013451095); Gm - Glycine max (XP_003554956); Pa - Prosopis alba (XP_028764818); At Arabidopsis thaliana (NP_197067). Fig. S4a. LIGAUT12 (alternative name LIIRX8) CDNA (GenBank accession number MW240679, 2276 bp) identified in yellow lupine (Lupinus luteus L.) and its deduced amino acid sequence [533 aa (ExPASy, translate tool), m.w. $=60.673 \mathrm{kD}$ and $\mathrm{pl}=8.94$ (ExPASy, ProtParam)]. The nucleotides are marked with lowercase letters, and amino acids with capital letters. The START and STOP codons (yellow background) are indicated. $5^{\prime}$ and $3^{\prime}$ UTR regions are marked with small italic letters before the ATG and after the TAG codons, respectively. The ORF is shown in pink. Fig. S4b. Maximum likelihood phylogenetic tree of 15 galacturonosyltransferase 12 (GAUT12) / irregular xylem 8 (IRX8) proteins (BlastP) with the highest degree of similarity to LIGAUT12/LIIRX8. Alignment and phylogenetic reconstructions were performed using the ETE3 V3.1.1 program as implemented in GenomeNet. The ML tree was inferred using PhyML v20160115. Branch supports are the Chi ${ }^{2}$-based parametric values returned by the approximate likelihood ratio test. Lalb- 
Lupinus albus (KAE9588601); Lang - Lupinus angustifolius (XP 019437125); Gm - Glycine max (XP_003543290); Ap - Abrus precatorius (XP_027341076); Pp - Prunus persica (XP_007213904); Pm - Prunus mume (XP_008244342); Ca - Cicer arietinum (XP_004487615); Ss - Spatholobus suberectus (TKY48985); CC - Cajanus cajan (XP 020235268); Ah - Arachis hypogaea (XP_025614714); Ad - Arachis duranensis (XP_015936483); Ai - Arachis ipaensis (XP_016170753); Md - Malus domestica (XP_008349951); Dz Durio zibethinus (XP_022716962); At - Arabidopsis thaliana (NP_200280). Fig. S4c. Multiple alignment (ClustalW) of 15 galacturonosyl transferase 12 (GAUT12) / irregular xylem 8 (IRX8) amino acid sequences (BlastP) that are closely related to LIGAUT12/LIIRX8. N-terminal cytoplasmic domain and the transmembrane domain (TM, yellow background) were predicted using the protein homology/analogy recognition engine V 2.0 (Phyre ${ }^{2}$ ) web portal. The specific glycosyl transferase family 8 (GT8) domain (pfam01501; black background) and the catalytic DxD motif (blue) were predicted using the CDD (NCBI). Fig. S4d. Comparison of GAUT12/IRX8 proteins (BlastP) derived from different plant species using the DiAlign program (Genomatix). For each pairwise alignment, the similarity (relative to the maximum similarity) and the number of identical amino acids (in $\%$ of shorter sequence) are given. Maximum values are underlined. The similarity value of 1.000 denotes only the two most similar sequences; it does not necessarily mean that these sequences are identical. Lalb - Lupinus albus (KAE9588601); Lang - Lupinus angustifolius (XP_019437125); Gm - Glycine max (XP_003543290); Ap - Abrus precatorius (XP_027341076); Pp Prunus persica (XP_007213904); Pm - Prunus mume (XP_008244342); Ca Cicer arietinum (XP_004487615); Ss - Spatholobus suberectus (TKY48985); Cc - Cajanus cajan (XP_020235268); Ah - Arachis hypogaea (XP_025614714); Ad - Arachis duranensis (XP_015936483); Ai - Arachis ipaensis (XP_016170753); Md - Malus domestica (XP_008349951); Dz Durio zibethinus (XP_022716962); At - Arabidopsis thaliana (NP_200280). Fig. S5a. LIPG/LIQRT2 CDNA (GenBank accession number MW240680, $1486 \mathrm{bp}$ ) identified in yellow lupine (Lupinus luteus L.) and its deduced amino acid sequence [428 aa (ExPASy, translate tool), m.w. $=47.623 \mathrm{kD}$ and $\mathrm{pl}=9.18$ (ExPASy, ProtParam)]. The nucleotides are marked with lowercase letters, and amino acids with capital letters. The START and STOP codons (yellow background) are indicated. 5' and 3' UTR regions are marked with small italic letters before the ATG and after the TGA codons. The ORF is shown in pink. Fig. S5b. Maximum likelihood phylogenetic tree of 19 polygalacturonases (PGs) (BlastP) with the highest degree of similarity to LIPG/LIQRT2. Alignment and phylogenetic reconstructions were performed using the ETE3 v3.1.1 program as implemented in GenomeNet. ML tree was inferred using PhyML v20160115. Branch supports are the $\mathrm{Chi}^{2}$-based parametric values returned by the approximate likelihood ratio test. Lang - Lupinus angustifolius (XP_019425177, PG-like); Lalb - Lupinus albus (KAE9590458, putative PG); Gm - Glycine max (XP_003545985, PG); Gs - Glycine soja (KHM99207, PG); Ca - Cicer arietinum (XP_004499889, PG QRT2); Ap - Abrus precatorius (XP_027343054, PG); Va Vigna angularis (XP_017424613, PG); Ad - Arachis duranensis (XP 015952899, PG QRT2-like); Ah - Arachis hypogaea (XP 025642914, PG QRT2); Ai - Arachis ipaensis (XP_020965356, PG); Mt - Medicago truncatula (XP 003595746, PG); Cc - Cajanus cajan (XP_020214988, PG); Cm - Cucurbita maxima (XP_022982564, PG); RC - Ricinus communis (XP_002517823, PG QRT2); Gh - Gossypium hirsutum (XP_016741024, PG QRT2-like); At Arabidopsis thaliana (O23147, ARABIDOPSIS DEHISCENCE ZONE POLYGALACTURONASE 1, ADPG1); A. thaliana (Q8RY29, ADPG2); A. thaliana (Q9SFB7, QUARTET2, QRT2); SI - Solanum lycopersicum (XP_004252072 PG QRT2). Fig. S5c. Multiple sequence alignment (ClustalW) of different polygalacturonases (PGs, BlastP) showing the highest similarity to LIPG/ LIQRT2. Four typical conserved domains of PGs, referred to as domains I, II, III and IV (RIKT) were indicatated in yellow, green, blue and red, respectively. A tyrosine $(Y)$ is marked in pink. 12 cysteine $(C)$ residues are marked in red letters. Three aspartic acids in NTD and DD structures (domains I and II, respectively) are marked with gray arrows. The histidine residue $(H)$ in domain III is marked with purple arrow. Fig. S5d. Comparison of PGs derived from different plant species using the DiAlign program (Genomatix). For each pairwise alignment, the similarity (relative to the maximum similarity) and the number of identical amino acids (in \% of shorter sequence) are given. Maximum values are underlined. The similarity value of 1.000 denotes only the two most similar sequences; it does not necessarily mean that these sequences are identical. Lang - Lupinus angustifolius (XP_019425177); Lalb - Lupinus albus (KAE9590458); Gm - Glycine max (XP_003545985); Gs - Glycine soja (KHM99207); Ca - Cicer arietinum (XP 004499889); Ap - Abrus precatorius (XP_027343054); Va - Vigna angularis (XP_017424613); Ad - Arachis duranensis (XP_015952899); AhArachis hypogaea (XP 025642914); Ai - Arachis ipaensis (XP_020965356); Mt - Medicago truncatula (XP_003595746); CC - Cajanus cajan (XP_020214988); Cm - Cucurbita maxima (XP_022982564); Rc - Ricinus communis (XP_002517823); Gh - Gossypium hirsutum (XP_016741024); At Arabidopsis thaliana (O23147, ADPG1); A. thaliana (Q8RY29, ADPG2); A. thaliana (Q9SFB7, QRT2); SI - Solanum lycopersicum (XP_004252072). Fig. S6a. LIPCS1L CDNA (GenBank accession number MW240681, 1773 bp) identified in yellow lupine (Lupinus luteus L.) and its deduced amino acid sequence [480 aa (ExPASy, translate tool), m.w. $=53.044 \mathrm{kD}$ and $\mathrm{pl}=5.42$ (ExPASy, ProtParam)]. The nucleotides are marked with lowercase letters, and amino acids with capital letters. The START and STOP codons (yellow background) are indicated. $5^{\prime}$ and $3^{\prime}$ UTR regions are marked with small italic letters before the ATG and after the TGA codons, resceptively. The ORF is shown in pink. Fig. S6b. Maximum likelihood phylogenetic tree of 15 PCS1 proteins (BlastP) with the highest degree of similarity to LIPCS1. Alignment and phylogenetic reconstructions were performed using the ETE3 V3.1.1 program as implemented in GenomeNet. The ML tree was inferred using PhyML v20160115. Branch supports are the Chi ${ }^{2}$-based parametric values returned by the approximate likelihood ratio test. Lang Lupinus angustifolius (XP_019412745); Vr - Vigna radiata (XP_014497350); Gm - Glycine max (XP_003530215); Mt - Medicago truncatula (XP_013448893); Ap - Abrus precatorius (XP_027349738); Cc - Cajanus cajan (XP_020206919); Vu - Vigna unguiculata (XP_027927273); Ca - Cicer arietinum (XP_004514393); Tp - Trifolium pratense (PNX74271); QI - Quercus lobata (XP_030925127); Pa - Prosopis alba (XP_028786489); St - Senna tora (KAF7825648); Qs - Quercus suber (XP_023913890); Tw - Tripterygium wilfordii (KAF5750924); At - Arabidopsis thaliana (OAO90380). Fig. S6c. Multiple alignment (ClustalW) of 15 PROMOTION OF CELL SURVIVAL1 (PCS1) amino acid sequences (BlastP) that are closely related to LIPCS1. Two motif in both N-terminal (DTGS) and C-terminal (DS/LGT) ends characteristic for pepsin like aspartic proteases are marked in yellow and light grey, respectivelly. Additionally, two catalytic residues (D) (white letters on red background) have been indicated. Active site flap ATLS and SSSS are marked green and light blue, respectively. Pepsin A like plant domain is shaded dark blue with white letters. TAXi_N domain (Xylanase inhibitor $\mathrm{N}$-terminal, sea color) and TAXi_C domain (violet) are additionally underlined. Lang - Lupinus angustifolius (XP_019412745); Vr - Vigna radiata (XP_014497350); Gm - Glycine max (XP_003530215); Mt - Medicago truncatula (XP_013448893); Ap - Abrus precatorius (XP_027349738); Cc - Cajanus cajan (XP_020206919); Vu - Vigna unguiculata (XP_027927273); Ca - Cicer arietinum (XP_004514393); Tp - Trifolium pratense (PNX74271); QI - Quercus lobata (XP_030925127); Pa - Prosopis alba (XP_028786489); St - Senna tora (KAF7825648); Qs - Quercus suber (XP_023913890); Tw - Tripterygium wilfordii (KAF5750924); At - Arabidopsis thaliana (OAO90380). Fig. S6d. Comparison of PCS1 proteins derived from different plant species using the DiAlign program (Genomatix). For each pairwise alignment, the similarity (relative to the maximum similarity) and the number of identical amino acids (in \% of shorter sequence) are given. Maximum values are underlined. The similarity value of 1.000 marks only the two most similar sequences; it does not necessarily mean that these sequences are identical. Lang - Lupinus angustifolius (XP_019412745); Vr - Vigna radiata (XP_014497350); Gm - Glycine max (XP_003530215); Mt - Medicago truncatula (XP_013448893); Ap - Abrus precatorius (XP_027349738); Cc - Cajanus cajan (XP_020206919); Vu - Vigna unguiculata (XP_027927273); Ca - Cicer arietinum (XP_004514393); Tp - Trifolium pratense (PNX74271); QI - Quercus lobata (XP 030925127); Pa - Prosopis alba (XP_028786489); St - Senna tora (KAF7825648); Qs - Quercus suber (XP_023913890); Tw - Tripterygium wilfordii (KAF5750924); At - Arabidopsis thaliana (OAO90380). Fig. S7a. L/GA30X cDNA (GenBank accession number MW240682, 1311 bp) identified in yellow lupine (Lupinus luteus L.) and its deduced amino acid sequence [320 aa (ExPASy, translate tool), m.w. $=36.576 \mathrm{kD}$ and pl $=5.67$ (ExPASy, ProtParam)]. The nucleotides are marked with lowercase letters, and amino acids with capital letters. The START and STOP codons (yellow background) are indicated. $5^{\prime}$ and $3^{\prime}$ UTR regions are marked with small italic letters before the ATG and after the TGA codons, resceptively. The ORF is shown in pink. Fig. S7b. Maximum likelihood phylogenetic tree of 10 
GA3ox proteins (BlastP) with the highest degree of similarity to LIGA30x. Alignment and phylogenetic reconstructions were performed using the ETE3 v3.1.1 program as implemented in GenomeNet. The ML tree was inferred using PhyML v20160115. Branch supports are the Chi ${ }^{2}$-based parametric values returned by the approximate likelihood ratio test. Lang Lupinus angustifolius (XP_019459798); Lalb - Lupinus albus (KAE9605408); Va - Vigna angularis (XP_017408358); Ss - Spatholobus suberectus (TKY63915); Gs - Glycine soja (KHN29357); VV - Vitis vinifera (XP_019072492); St - Solanum tuberosum (XP_006339568); Ns - Nicotiana sylvestris (XP_009779750); Na - Nicotiana attenuata (XP_019262262); At -Arabidopsis thaliana (NP_178149). Fig. S7c. Multiple alignment (ClustalW) of 10 GIBBERELLIN 3-OXIDASE (GA3ox) amino acid sequences (BlastP) that are closely related to LIGA3ox. Gibberellin 3- $\beta$-dioxygenase domain (black background) and domain characteristic for 2-oxoglutarate (2OG) and Fe (II)-dependent oxygenase (Oxy) superfamily (blue background) are indicated. The putative His- $x-A s p-(x) n-H i s(H \times D \ldots H)$ and Arg-x-Ser (RxS) motif locations are highlighted in red and green, respectively. Domains and specific, highly conserved motifs were delineated from the CDD (NCBI) and [53]. Lang - Lupinus angustifolius (XP_019459798); Lalb - Lupinus albus (KAE9605408); Va - Vigna angularis (XP_017408358); Ss - Spatholobus suberectus (TKY63915); Gs - Glycine soja (KHN29357); VV - Vitis vinifera (XP_019072492); St - Solanum tuberosum (XP_006339568); Ns - Nicotiana sylvestris (XP_009779750); Na - Nicotiana attenuata (XP_019262262); At -Arabidopsis thaliana (NP_178149). Fig. S7d. Comparison of GIBBERELLIN 3-OXIDASES (GA3Oxs) derived from different plant species using the DiAlign program (Genomatix). For each pairwise alignment, the similarity (relative to the maximum similarity) and the number of identical amino acids (in \% of shorter sequence) are given. Maximum values are underlined. The similarity value of 1.000 marks only the two most similar sequences; it does not necessarily mean that these sequences are identical. Lang - Lupinus angustifolius (XP_019459798); Lalb - Lupinus albus (KAE9605408); Va - Vigna angularis (XP_017408358); Ss - Spatholobus suberectus (TKY63915); Gs - Glycine soja (KHN29357); VV - Vitis vinifera (XP_019072492); St - Solanum tuberosum (XP_006339568); Ns - Nicotiana sylvestris (XP_009779750); Na - Nicotiana attenuata (XP_019262262); At -Arabidopsis thaliana (NP_178149). Fig. S8a. LIGA20x1 CDNA (GenBank accession number MG181996, 1458 bp) identified in yellow lupine (Lupinus luteus L.) and its deduced amino acid sequence [330 aa (ExPASy, translate tool), m.w. $=37.005 \mathrm{kD}$ and $\mathrm{pl}=8.14$ (ExPASy, ProtParam)]. The nucleotides are marked with lowercase letters, and amino acids with capital letters. The START and STOP codons (yellow background) are indicated. 5' and 3' UTR regions are marked with small italic letters before the ATG and after the TGA codons, resceptively. The ORF is shown in pink. Fig. S8b. Maximum likelihood phylogenetic tree of 15 GIBBERELLIN 2OXIDASES (GA2oxs, BlastP) with the highest degree of similarity to LIGA20x1. Alignment and phylogenetic reconstructions were performed using the ETE3 v3.1.1 as implemented in GenomeNet. The ML tree was inferred using PhyML v20160115. Branch supports are the Chi ${ }^{2}$-based parametric values returned by the approximate likelihood ratio test. Lang - Lupinus angustifolius (XP_019424643); Lalb - Lupinus albus (KAE9590556); Mp - Mucuna pruriens (RDX87676); Ss - Spatholobus suberectus (TKY49301); Ap - Abrus precatorius (XP_027342450); Va - Vigna angularis (NP_001316752); CC - Cajanus cajan (XP_020204195); Vu - Vigna unguiculata (XP_027926310); Gm - Glycine max (XP_003543155); Ps - Pisum sativum (AADD45425); Gs - Glycine soja (XP_028201454); Ca - Cicer arietinum (XP_004488652); Jr - Juglans regia (XP_018824979); Nt - Nicotiana tabacum (XP_016500243); At - Arabidopsis thaliana (Q8LEA2). Fig. S8c. Multiple alignment (ClustalW) of 15 GIBBERELLIN 2-OXIDASE (GA2ox) amino acid sequences (BlastP) that are closely related to LIGA20x1. Gibberellin 2$\beta$-dioxygenase domain (black background) and domain characteristic for 2-oxoglutarate (2OG)-Fe (II)-dependent oxygenase superfamily (blue background) are indicated. Both domains designated using the CDD (NCBI). Additionally, amino acid residues presumed to bind $\mathrm{Fe}^{2+}$ at the active site of protein are indicated with red $(H \times D \ldots H)$ and green $(R \times S)$ letters. Fig. S8d. Comparison of GIBBERELLIN 2-OXIDASES (GA2oxs) derived from different plant species using the DiAlign program (Genomatix). For each pairwise alignment, the similarity (relative to the maximum similarity) and the number of identical amino acids (in \% of shorter sequence) are given. Maximum values are underlined. The similarity value of 1.000 marks only the two most similar sequences; it does not necessarily mean that these sequences are identical. Lang - Lupinus angustifolius (XP_019424643); Lalb - Lupinus albus (KAE9590556); Mp - Mucuna pruriens (RDX87676); Ss Spatholobus suberectus (TKY49301); Ap - Abrus precatorius (XP_027342450); Va - Vigna angularis (NP_001316752); Cc - Cajanus cajan (XP_020204195); Vu - Vigna unguiculata (XP_027926310); Gm - Glycine max (XP_003543155); Ps - Pisum sativum (AAD45425); Gs - Glycine soja (XP_028201454); Ca - Cicer arietinum (XP_004488652); Jr - Juglans regia (XP_018824979); Nt - Nicotiana tabacum (XP_016500243); At - Arabidopsis thaliana (Q8LEA2). Fig. S9a. LIGAMYB CDNA (GenBank accession number MW240675, 2038 bp) identified in yellow lupine (Lupinus luteus L.) and its deduced amino acid sequence [533 aa (ExPASy, translate tool), m.w. = $59.048 \mathrm{kD}$ and $\mathrm{pl}=5.25$ (ExPASy, ProtParam)]. The nucleotides are marked with lowercase letters, and amino acids with capital letters. The START and STOP codons (yellow background) are indicated. 5' and 3' UTR regions are marked with small italic letters before the ATG and after the TGA codons, resceptively. The ORF is shown in pink. 21 nucleotide sequence in blue background corresponds to target site for LI-miR159. Fig. s9b. Maximum likelihood phylogenetic tree of 16 GAMYB proteins (BlastP) with the highest degree of similarity to LIGAMYB. Alignment and phylogenetic reconstructions were performed using the ETE3 v3.1.1 as implemented in GenomeNet. The ML tree was inferred using PhyML v20160115. Branch supports are the Chi ${ }^{2}$-based parametric values returned by the approximate likelihood ratio test. Lang - Lupinus angustifolius (XP_019449331); Lalb - Lupinus albus (KAE9617167); Ss - Spatholobus suberectus (TKY68413); Cc - Cajanus cajan (XP_020219565); Ap - Abrus precatorius (XP_027354365); Mp - Mucuna pruriens (RDX95167); Gm - Glycine max (AHB19229); Gs - Glycine soja (XP_028187659); Ah - Arachis hypogaea (XP_025615172); Ai - Arachis ipaensis (XP_016171706); Ad - Arachis duranensis (XP_015937001); At - Arabidopsis thaliana (AAS10086, AtMYB33), (AAS10055, AtMYB65), (NP_194423, AtMYB97), (NP_001077993, AtMYB101), (NP_568819, AtMYB120). Fig. S9c. Multiple alignment (ClustalW) of $16 \mathrm{MYB}$ amino acid sequences (BlastP) that are closely related to LIGAMYB. The R2R3 domain near the $5^{\prime}$ terminus (black) and Box 1 (yellow), Box 2 (green), Box 3 (blue) domains distributed throughout the protein were designated. REB1 domain characteristic for Myb superfamily proteins (underlined), Myb_DNA-binding domain (pink) and SANT (SWI3, ADA2, N-CoR and TFIIIB" DNA-binding) domain (gray) have been designated using the CDD (NCBI). Lang - Lupinus angustifolius (XP_019449331); Lalb - Lupinus albus (KAE9617167); Ss - Spatholobus suberectus (TKY68413); CC - Cajanus cajan (XP_020219565); Ap - Abrus precatorius (XP_027354365); Mp - Mucuna pruriens (RDX95167); Gm - Glycine max (AHB19229); Gs - Glycine soja (XP_028187659); Ah - Arachis hypogaea (XP_025615172); Ai - Arachis ipaensis (XP_016171706); Ad - Arachis duranensis (XP_015937001); At - Arabidopsis thaliana (AAS10086, AtMYB33), (AAS10055, AtMYB65), (NP_194423, AtMYB97), (NP_001077993,

AtMYB101), (NP_568819, AtMYB120). Fig. S9d. Comparison of GAMYB proteins derived from different plant species using the DiAlign program (Genomatix). For each pairwise alignment, the similarity (relative to the maximum similarity) and the number of identical amino acids (in \% of shorter sequence) are given. Maximum values are underlined. The similarity value of 1.000 marks only the two most similar sequences; it does not necessarily mean that these sequences are identical. Lang - Lupinus angustifolius (XP_019449331); Lalb - Lupinus albus (KAE9617167); Ss - Spatholobus suberectus (TKY68413); CC - Cajanus cajan (XP_020219565); Ap - Abrus precatorius (XP_027354365); Mp - Mucuna pruriens (RDX95167); Gm - Glycine max (AHB19229); Gs - Glycine soja (XP_028187659); Ah - Arachis hypogaea (XP_025615172); Ai - Arachis ipaensis (XP_016171706); Ad - Arachis duranensis (XP_015937001); At - Arabidopsis thaliana (AAS10086, AtMYB33), (AAS10055, AtMYB65), (NP_194423, AtMYB97), (NP_001077993, AtMYB101), (NP_568819, AtMYB120). Fig. S10. The cDNA sequence of $L /-$ MIR159 identified in yellow lupine (GenBank accession number MW240683). The 21-nucleotide fragment that constitutes the mature miR159 is marked in yellow. Fig. S11. The domain structures of LICAD (cinnamyl alcohol dehydrogenase), LICesA8/LIIRX1 (cellulose synthase A catalytic subunit 8/IRREGULAR XYLEM1), LICOBL4/IRX6 (COBRA-like4), LIGAUT12/LIIRX8 (galacturonosyltransferase 12), LIPG/LIQRT2 (polygalacturonase/ QUARTET2) and LIPCS1 (PROMOTION OF CELL SURVIVAL1) identified in yellow lupine (Lupinus luteus L.). (A-F) The predicted tertiary 3D models constructed by the ROBETTA protein modelling server visualized by the ChimeraX program. (A'-F') Conserved domains, motifs and 
specific amino acids identified via different tools described in the Methods section. The colours used in the 3D protein models correspond to the colours presented in the protein diagrams. The specific functions of domains/motifs/amino acids are described in detail in Table 1 (main manuscript). Fig. S12. The domain structures of LIGA3ox (gibberellin 3oxidase), LIGA20x1 (gibberellin 2-oxidase1) and LIGAMYB identified in yellow lupine (Lupinus luteus $\mathrm{L}$.). (A-C) The predicted tertiary 3D models constructed by the ROBETTA protein modelling server visualized by the ChimeraX program. ( $\left.A^{\prime}-C^{\prime}\right)$ Conserved domains, motifs and specific amino acids identified via different tools described in the Method section. The colours used in the 3D protein models correspond to the colours presented in the protein diagrams. The specific functions of domains/motifs/ amino acids are described in detail in Table 1 (main manuscript). Fig. S13. (A) Image of the electrophoretic separation of the PCR product carried out with degenerate primers to identify a CDNA fragment of LIGAMYB in yellow lupine on a 1.2\% agarose gel in $0,5 \times$ TBE buffer at $5 \mathrm{~V} / \mathrm{cm}$ in the presence of the GeneRuler $100 \mathrm{bp}$ DNA ladder marker ( $M$, Fermentas); (B) Simplified diagram of the pSC-A-amp/kan vector (Agilent Technologies); (C) Representative Petri dish containing S-Gal/LB agar blend with one shot Mach1-T1 E. coli. White bacterial colonies took the recombinant form of the pCRII-TOPO vector, while dark blue bacterial colonies took the vector without the insert. (D) Image of electrophoretic separation of plasmid DNA digested with the restriction enzyme EcoRl (Fermentas) to confirm the presence of the insert - the CDNA fragment of LIGAMYB. Separation was performed on a $1.2 \%$ agarose gel in $0.5 \times$ TBE buffer at $5 \mathrm{~V} / \mathrm{cm}$ and in the presence of a GeneRuler $100 \mathrm{bp}$ DNA ladder marker $(M)$. The arrow indicates the sample that was sent for sequencing (Genomed, Warsaw, Poland). Fig. S14. (A, B) Electrophoretic separation images of the PCR products ( $A$, the results obtained with the first pair of primers, specific + degenerate) and 3'RACE-PCR (B, the results obtained with the second pair of primers) for LIGAMyb in $1.2 \%$ agarose gels in $0.5 \times$ TBE buffer at $5 \mathrm{~V} / \mathrm{cm}$ in the presence of GeneRuler $100 \mathrm{bp}$ DNA Ladder marker ( $M$, Fermentas); (C, D) Electrophoretic separation images of plasmid DNA containing inserts of various sizes that were digested with the restriction enzyme EcoRl (Fermentas) on 1.2\% agarose gels in $0.5 \times$ TBE buffer at $5 \mathrm{~V} / \mathrm{cm}$ and in the presence of GeneRuler $100 \mathrm{bp}$ DNA Ladder marker $(M)$; Transformation results obtained with the reaction products carried out using the first pair of primers $(C)$ and the second pair of primers (D); arrows indicate the individual samples that were sent for sequencing (Genomed, Warsaw, Poland). Fig. S15. The negative control required for validation of the immunohistochemical reactions was carried out by omitting incubation with the primary antibody, and showed no labelling. The autofluorescence signal of the cell walls and pollen grains is visible. DAPI was used to stain cell nuclei. Scale bar $=50 \mu \mathrm{m}$. Tab. S1. (A) Sequences of degenerate primers designed based on the CDNA sequences of GAMYB genes derived from different plant species (BlastP); (B) Sequences of specific, degenerate and universal primers used in 3' RACE (rapid amplification of CDNA ends)-PCR; GSOP - gene specific outer primer, OP - outer primer, GSIP - gene specific inner primer, IP - inner primer; (C) Sequences of specific primers, their melting temperature (Tm) and UPL (UNIVERSAL PROBE LIBRARY) probe sequences and numbers used in GPCR for all studied genes and LIACT. The length of the amplified product in PCR and the annealing temperature of the primers are also given. (PPTX 19524 kb)

\section{Acknowledgements}

The authors would like to thank Michał Świdziński (Nicolaus Copernicus University, Faculty of Biological and Veterinary Sciences, Department of Cellular and Molecular Biology) for helping with the preparation of ultratinsections for microscopy experiments.

\section{Authors' contributions}

Conception and design, KM; Investigations, KM and KP; Analyses, KM; Writing the manuscript, KM; Preparing the figures and tables, KM and KP; Supervision, KM. The authors read and approved the final manuscript.

\section{Funding}

This research was supported by the Polish Ministry of Agriculture and Rural Development [Grant Numbers 149/2011 and 222/2015].

\section{Availability of data and materials}

The data supporting the conclusions of this article are included within the article and its additional files. The CDNA sequences of all identified genes of yellow lupine are available at GenBank (NCBI) under accession numbers: MW240675, MW240676, MW240677, MW240678, MW240679, MW240680, MW240681, MW240682, MW240683 and MG181996. The nucleotide and protein sequences were downloaded from GenBank and Protein database (NCBI), respectively. The RNA-Seq data are available at the NCBI in the Sequence Read Archive (SRA) database under accession number PRJNA285604 (BioProject) and experiment accession number SRX1069734.

\section{Declarations}

Ethics approval and consent to participate

Not applicable.

\section{Consent for publication}

Not applicable.

\section{Competing interests}

The authors declare that they have no competing interests.

Received: 26 November 2020 Accepted: 4 June 2021

Published online: 02 July 2021

\section{References}

1. Sanders PM, Bui AQ, Weterings K, Mclntire KN, Hsu YC, Lee PY, et al. Anther developmental defects in Arabidopsis thaliana male-sterile mutants. Sex Plant Reprod. 1999;11(6):297-322. https://doi.org/10.1007/s004970050158.

2. Scott RJ, Spielman M, Dickinson HG. Stamen structure and function. Plant Cell. 2004;16:46-60.

3. Wilson ZA, Zhang DB. From Arabidopsis to rice: pathways in pollen development. J Exp Bot. 2009;60(5):1479-92. https://doi.org/10.1093/jxb/ erp095.

4. Wilson ZA, Song J, Taylor B, Yang C. The final split: the regulation of anther dehiscence. J Exp Bot. 2011;62(5):1633-49. https://doi.org/10.1093/jxb/ erro14.

5. Marciniak K, Przedniczek K. Comprehensive insight into gibberellin- and jasmonate-mediated stamen development. Genes. 2019;10(10):811. https:// doi.org/10.3390/genes10100811.

6. Goldberg RB, Beals TP, Sanders PM. Anther development: basic principles and practical applications. Plant Cell. 1993;5(10):1217-29. https://doi.org/1 $0.1105 /$ tpc.5.10.1217

7. Mandaokar A, Thines B, Shin B, Lange BM, Choi G, Koo YJ, et al. Transcriptional regulators of stamen development in Arabidopsis identified by transcriptional profiling. Plant J. 2006;46(6):984-1008. https://doi.org/1 0.1111/j.1365-313X.2006.02756.x.

8. Sanders PM, Bui AQ, Le BH, Goldberg RB. Differentiation and degeneration of cells that play a major role in tobacco anther dehiscence. Sex Plant Reprod. 2005;17(5):219-41. https://doi.org/10.1007/s00497-004-0231-y.

9. Sanders PM, Lee PY, Biesgen C, Boone JD, Beals TP, Weiler EW, et al. The Arabidopsis DELAYED DEHISCENCE1 gene encodes an enzyme in the jasmonic acid synthesis pathway. Plant Cell. 2000;12(7):1041-61. https://doi. org/10.1105/tpc.12.7.1041.

10. Matsui T, Omasa K, Horie T. Mechanism of anther dehiscence in rice (Oryza sativa L.). Ann Bot. 1999;84(4):501-6. https://doi.org/10.1006/anbo.1999.0943.

11. Garcia CC. An approach to the diversity of endothecial thickenings in Solanaceae. Flora. 2002:197(3):214-23. https://doi.org/10.1078/0367-2530-00032.

12. Garcia CC. Anther wall formation in Solanaceae species. Ann Bot. 2002;90(6): 701-6. https://doi.org/10.1093/aob/mcf248.

13. Boerjan W, Ralph J, Baucher M. Lignin biosynthesis. Annu Rev Plant Biol. 2003;54(1):519-46. https://doi.org/10.1146/annurev.arplant.54.031902.134938.

14. Thevenin J, Pollet B, Letarnec B, Saulnier L, Gissot L, Maia-Grondard A, et al. The simultaneous repression of CCR and CAD, two enzymes of the lignin biosynthetic pathway, results in sterility and dwarfism in Arabidopsis thaliana. Mol Plant. 2011;4(1):70-82. https://doi.org/10.1093/mp/ssq045.

15. Brown DM, Zeef LA, Ellis J, Goodacre R, Turner SR. Identification of nove genes in Arabidopsis involved in secondary cell wall formation using expression profiling and reverse genetics. Plant Cell. 2005;17(8):2281-95. https://doi.org/10.1105/tpc.105.031542. 
16. Taylor NG, Scheible WR, Cutler S, Somerville CR, Turner SR. The irregular xylem3 locus of Arabidopsis encodes a cellulose synthase required for secondary cell wall synthesis. Plant Cell. 1999;11(5):769-80. https://doi.org/1 0.1105/tpc.11.5.769.

17. Taylor NG, Howells RM, Huttly AK, Vickers K, Turner SR. Interactions among three distinct CesA proteins essential for cellulose synthesis. Proc Natl Acad Sci U S A. 2003;100(3):1450-5. https://doi.org/10.1073/pnas.0337628100.

18. Hao Z, Avci U, Tan L, Zhu X, Glushka J, Pattathil S, et al. Loss of Arabidopsis GAUT12/IRX8 causes anther indehiscence and leads to reduced $G$ lignin associated with altered matrix polysaccharide deposition. Front Plant Sci. 2014:5:357.

19. Ogawa M, Kay P, Wilson S, Swain SM. ARABIDOPSIS DEHISCENCE ZONE POLYGALACTURONASE1 (ADPG1), ADPG2, and QUARTET2 are polygalacturonases required for cell separation during reproductive development in Arabidopsis. Plant Cell. 2009;21(1):216-33. https://doi.org/1 $0.1105 /$ tpc.108.063768.

20. Kuriyama H, Fukuda $\mathrm{H}$. Developmental programmed cell death in plants. Curr Opin Plant Biol. 2002;5(6):568-73. https://doi.org/10.1016/S1369-52 66(02)00305-9.

21. Ge X, Dietrich C, Matsuno M, Li G, Berg H, Xia Y. An Arabidopsis aspartic protease functions as an anti-cell-death component in reproduction and embryogenesis. EMBO Rep. 2005;6(3):282-8. https://doi.org/10.1038/sj. embor.7400357.

22. Feys BJ, Benedetti CE, Penfold CN, Turner JG. Arabidopsis mutants selected for resistance to the phytotoxin coronatine are male sterile, insensitive to methyl jasmonate, and resistant to a bacterial pathogen. Plant Cell. 1994; 6(5):751-9. https://doi.org/10.2307/3869877.

23. Ishiguro S, Kawai-Oda A, Ueda J, Nishida I, Okada K. The DEFECTIVE IN ANTH ER DEHISCHENCE1 gene encodes a novel phospholipase A1 catalyzing the initial step of jasmonic acid biosynthesis, which synchronizes pollen maturation, anther dehiscence, and flow opening in Arabidopsis. Plant Cell. 2001;13(10):2191-209. https://doi.org/10.1105/tpc.010192.

24. Cheng H, Qin L, Lee S, Fu X, Richards DE, Cao D, et al. Gibberellin regulates Arabidopsis floral development via suppression of DELLA protein function. Development. 2004;131(5):1055-64. https://doi.org/10.1242/dev.00992.

25. Chhun T, Aya K, Asano K, Yamamoto E, Morinaka Y, Watanabe M, et al. Gibberellin regulates pollen viability and pollen tube growth in rice. Plant Cell. 2007;19(12):3876-88. https://doi.org/10.1105/tpc.107.054759.

26. Hu J, Mitchum MG, Barnaby N, Ayele BT, Ogawa M, Nam E, et al. Potential sites of bioactive gibberellin production during reproductive growth in Arabidopsis. Plant Cell. 2008;20(2):320-36. https://doi.org/10.1105/tpc.107.057752.

27. Rieu I, Ruiz-Rivero O, Fernandez-Garcia N, Griffiths J, Powers SJ, Gong F, et al. The gibberellin biosynthetic genes AtGA200x1 and AtGA200x2 act, partially redundantly, to promote growth and development throughout the Arabidopsis life cycle. Plant J. 2008;53(3):488-504. https://doi.org/10.1111/ j.1365-313X.2007.03356.x

28. Aya K, Ueguchi-Tanaka M, Kondo M, Hamada K, Yano K, Nishimura M, et al. Gibberellin modulates anther development in rice via the transcriptional regulation of GAMYB. Plant Cell. 2009;21(5):1453-72. https://doi.org/10.1105/ tpc.108.062935.

29. Murray F, Kalla R, Jacobsen J, Gubler F. A role for HvGAMYB in anther development. Plant J. 2003;33(3):481-91. https://doi.org/10.1046/j.1365-313 X.2003.01641.X.

30. Achard P, Herr A, Baulcombe DC, Harberd NP. Modulation of floral development by a gibberellin-regulated microRNA. Development. 2004: 131(14):3357-65. https://doi.org/10.1242/dev.01206.

31. Zheng L, Nagpal P, Villarino G, Trinidad B, Bird L, Huang Y, et al. miR167 limits anther growth to potentiate anther dehiscence. Development. 2019; 146:174375.

32. Glazinska P, Wojciechowski W, Kulasek M, Glinkowski W, Marciniak K, Klajn N, et al. De novo transcriptome profiling of flowers, flower pedicels and pods of Lupinus luteus (yellow lupine) reveals complex expression changes during organ abscission. Front Plant Sci. 2017;8:641. https://doi.org/10.3389/fpls.2017.00641.

33. Marciniak K, Kućko A, Wilmowicz E, Świdziński M, Przedniczek K, Kopcewicz J. Gibberellic acid affects the functioning of the flower abscission zone in Lupinus luteus via cooperation with the ethylene precursor independently of abscisic acid. J Plant Physiol. 2018;229:170-4. https://doi.org/10.1016/j.jplph.2 018.07.014.

34. Marciniak K, Przedniczek K. Gibberellin signaling repressor LIDELLA1 controls the flower and pod development of yellow lupine (Lupinus luteus L.). Int J Mol Sci. 2020;21(5):1815. https://doi.org/10.3390/ijms21051815.
35. Kućko A, Smoliński D, Wilmowicz E, Florkiewicz A, de Dios Alché J. Spatiotemporal localization of $\angle I B O P$ following early events of floral abscission in yellow lupine. Protoplasma. 2019;256(5):1173-83. https://doi.org/10.1007/ s00709-019-01365-3.

36. Bonawitz ND, Chapple $C$. The genetics of lignin biosynthesis: connecting genotype to phenotype. Annu Rev Genet. 2010;44(1):337-63. https://doi. org/10.1146/annurev-genet-102209-163508.

37. Taylor NG, Laurie S, Turner SR. Multiple cellulose synthase catalytic subunits are required for cellulose synthesis in Arabidopsis. Plant Cell. 2000;12(12): 2529-39. https://doi.org/10.1105/tpc.12.12.2529.

38. Galeano E, Vasconcelos TS, Carrer H. Characterization of Cinnamyl Alcohol Dehydrogenase gene family in lignifying tissues of Tectona grandis L. Silvae Genet. 2018;67(1):1-11. https://doi.org/10.2478/sg-2018-0001.

39. Tang $R$, Zhang XQ, Li YH, Xie XM. Cloning and in silico analysis of a cinnamyl alcohol dehydrogenase gene in Pennisetum purpureum. J Genet. 2014;93(1):145-58. https://doi.org/10.1007/s12041-014-0355-2.

40. Jin Y, Zhang C, Liu W, Qi H, Chen H, Cao S. The cinnamyl alcohol dehydrogenase gene family in melon (Cucumis melo L.): bioinformatic analysis and expression patterns. PLoS One. 2014;9(7):e101730. https://doi. org/10.1371/journal.pone.0101730.

41. Richmond T. Higher plant cellulose synthases. Genome Biol. 2000;1:3001.1-6.

42. Hill JL Jr, Josephs C, Barnes WJ, Anderson CT, Tien M. Longevity in vivo of primary cell wall cellulose synthases. Plant Mol Biol. 2018;96(3):279-89. https://doi.org/10.1007/s11103-017-0695-4.

43. Olek AT, Rayon C, Makowski L, Kim HR, Ciesielski P, Badger J, et al. The structure of the catalytic domain of a plant cellulose synthase and its assembly into dimers. Plant Cell. 2014;26(7):2996-3009. https://doi.org/10.11 05/tpc.114.126862

44. Slabaugh E, Davis JK, Haigler CH, Yingling YG, Zimmer J. Cellulose synthases: new insights from crystallography and modeling. Trends Plant Sci. 2014; 19(2):99-106. https://doi.org/10.1016/j.tplants.2013.09.009.

45. Sindhu A, Langewisch T, Olek A, Multani DS, McCann MC, Vermerris W. Maize Brittle stalk2 encodes a COBRA-like protein expressed in early organ development but required for tissue flexibility at maturity. Plant Physiol. 2007;145(4):1444-59. https://doi.org/10.1104/pp.107.102582

46. Gao Y, Bian L, Shi J, Xu J, Xi M, Wang G. Expression of a conifer COBRA-like gene CICOBL1 from Chinese fir (Cunninghamia lanceolata) alters the leaf architecture in tobacco. Plant Physiol Biochem. 2013;70:483-91. https://doi. org/10.1016/j.plaphy.2013.06.013.

47. Bussink HJD, Buxton FP, Visser J. Expression and sequence comparison of the Aspergillus niger and Aspergillus tubigensis genes encoding polygalacturonase II. Curr Genet. 1991;19(6):467-74. https://doi.org/10.1007/ BF00312738.

48. Tebbutt SJ, Rogers HJ, Lonsdale DM. Characterization of a tobacco gene encoding a pollen-specific polygalacturonase. Plant Mol Biol. 1994;25(2): 283-97. https://doi.org/10.1007/BF00023244

49. Torki M, Mandaron $P$, Mache R, Falconet D. Characterization of a ubiquitous expressed gene family encoding polygalacturonase in Arabidopsis thaliana. Gene. 2000;242(1-2):427-36. https://doi.org/10.1016/S0378-1119(99)00497-7.

50. Rexová-Benková L. Evidence for the role of carboxyl groups in activity of endopolygalacturonase of Aspergillus niger. Chemical modification by carbodiimide reagent. Collect Czechoslov Chem Commun. 1990;55(5):138995. https://doi.org/10.1135/cccc19901389.

51. Rao MN, Kembhavi AA, Pant A. Implication of tryptophan and histidine in the active site of endo-polygalacturonase from Aspergillus ustus: elucidation of the reaction mechanism. Biochim Biophys Acta. 1996;1296(2):167-73. https://doi.org/10.1016/0167-4838(96)00067-2.

52. Stratilová E, Dzúrová $M$, Markovic $O$, Jörnvall $H$. An essential tyrosine residue of Aspergillus polygalacturonase. FEBS Lett. 1996;382(1-2):164-6. https://doi. org/10.1016/0014-5793(96)00146-9.

53. Lee DJ, Zeevaart JAD. Differential regulation of RNA levels of gibberellin dioxygenases by photoperiod in spinach. Plant Physiol. 2003;130:2085-94.

54. Bonner LJ, Dickinson HG. Anther dehiscence in Lycopersicon esculentum mill. I Structural aspects. New Phytol. 1989;113(1):97-115. https://doi.org/10.1111/ j.1469-8137.1989.tb02399.x

55. Fernandez J, Wilson ZA. Non-destructive staging of barley reproductive development for molecular analysis based upon external morphology. J Exp Bot. 2012;63:4085-94.

56. Browne RG, lacuone S, Li SF, Dolferus R, Parish RW. Anther morphological development and stage determination in Triticum aestivum. Front Plant Sci. 2018;9:228. https://doi.org/10.3389/fpls.2018.00228. 
57. Keijzer CJ, Leferinkten Klooster HB, Reinders MC. The mechanics of the grass flower: anther dehiscence and pollen shedding in maize. Ann Bot. 1996; 78(1):15-21. https://doi.org/10.1006/anbo.1996.0089.

58. Garcia CC, Nepi M, Pacini E. Structural aspects and ecophysiology of anther opening in Allium triquetrum. Ann Bot. 2006;97(4):521-7. https://doi.org/10.1 093/aob/mcl015.

59. Lord EM. Cleistogamy: a tool for the study of floral morphogenesis, function and evolution. Bot Rev. 1981;47(4):421-49. https://doi.org/10.1007/BF02860538.

60. Forbes I, Leuck DB, Edwardson JR, Burns RE. Natural cross-pollination in blue lupine (Lupinus angustifolius L.) in Georgia and Florida. Crop Sci. 1971;11(6): 851-4. https://doi.org/10.2135/cropsci1971.0011183X001100060025x.

61. Smith BW. Arachis hypogaea. Aerial flower and subterranean fruit. Am J Bot. 1950;37(10):802-15. https://doi.org/10.1002/j.1537-2197.1950.tb11073.x.

62. Chichiricco G. Ripening and dehiscence of the anther in some Crocus (Iridaceae) species. Caryologia. 2000;53(3-4):255-60. https://doi.org/10.1080/ 00087114.2000 .10589203

63. Cheng PC, Greyson RI, Walden DB. Comparison of anther development in genic-male-sterile and in male-fertile corn (Zea mays) from light microscopy and scanning microscopy. Can J Bot. 1979:57(6):578-96. https://doi.org/1 0.1139/b79-076

64. Bianchini M, Pacini L. Explosive anther dehiscence in Ricinus communis involves cell wall modifications and relative humidity. Int J Plant Sci. 1996; 157(6):739-45. https://doi.org/10.1086/297397.

65. Niu E, Shang X, Cheng C, Bao J, Zeng Y, Cai C, et al. Comprehensive analysis of the COBRA-Like (COBL) gene family in Gossypium identifies two COBLs potentially associated with fiber quality. PLoS One. 2015;10(12):e0145725. https://doi.org/10.1371/journal.pone.0145725.

66. Jung KW, Oh SI, Kim YY, Yoo KS, Cui MH, Shin JS. Arabidopsis histidinecontaining phosphotransfer factor 4 (AHP4) negatively regulates secondary wall thickening of the anther endothecium during flowering. Mol Cells. 2008;25(2):294-300.

67. Gorguet B, Schipper D, van Lammeren A, Visser RG, van Heusden AW. ps-2, the gene responsible for functional sterility in tomato, due to non-dehiscent anthers, is the result of a mutation in a novel polygalacturonase gene. Theor Appl Genet. 2009;118(6):1199-209. https://doi.org/10.1007/s00122009-0974-9.

68. Ye J, Yang X, Yang Z, Niu F, Chen Y, Zhang L, Song X. Comprehensive analysis of polygalacturonase gene family highlights candidate genes related to pollen development and male fertility in wheat (Triticum aestivum L.). Planta 2020;252:31, 2, DOl: https://doi.org/10.1007/s00425-020-03435-w.

69. Lyu M, Iftikhar J, Guo R, Wu B, Cao J. Patterns of expansion and expression divergence of the polygalacturonase gene family in Brassica oleracea. Int J Mol Sci. 2020;21(16):5706. https://doi.org/10.3390/ijms21165706.

70. Keijzer $C$. The processes of anther dehiscence and pollen dispersal. I. the opening mechanism of longitudinally dehiscing anthers. New Phytol. 1987; 105(3):487-9. https://doi.org/10.1111/j.1469-8137.1987.tb00886.x.

71. Plackett ARG, Thomas SG, Wilson ZA, Hedden P. Gibberellin control of stamen development: a fertile field. Trends Plant Sci. 2011;16(10):568-78. https://doi.org/10.1016/j.tplants.2011.06.007.

72. Kobayashi M, Yamaguchi I, Murofushi N, Ota Y, Takahashi N. Fluctuation and localization of endogenous gibberellins in rice. Agri Biol Chem. 1988:52: 1189-94.

73. Izhaki A, Borochov A, Zamski E, Weiss D. Gibberellin regulates postmicrosporogenesis processes in petunia anthers. Physiol Plant. 2002;115(3) 442-7. https://doi.org/10.1034/j.1399-3054.2002.1150314x.

74. Weiss D, Halevy AH. Stamens and gibberellin in the regulation of corolla pigmentation and growth in Petunia hybrida. Planta. 1989;179(1):89-96. https://doi.org/10.1007/BF00395775.

75. Itoh H, Ueguchi-Tanaka M, Kawaide H, Chen X, Kamiya Y, Matsuoka M. The gene encoding tobacco gibberellin $3 \beta$-hydroxylase is expressed at the site of GA action during stem elongation and flower organ development. Plant J. 1999;20(1):15-24. https://doi.org/10.1046/j.1365-313X.1999.00568.x.

76. Itoh H, Ueguchi-Tanaka M, Sentoku N, Kitano H. Matsuoka M, Kobayashi M. Cloning and functional analysis of two gibberellin $3 \beta$-hydroxylase genes that are differently expressed during the growth of rice. Proc Natl Acad Sci U S A 2001;98:8909-8914, 15, DOl: https://doi.org/10.1073/pnas.141239398.

77. Gocal GF, Sheldon CC, Gubler F, Moritz T, Bagnall DJ, MacMillan CP, et al. GAMYB-like genes, flowering, and gibberellin signaling in Arabidopsis. Plant Physiol. 2001;127(4):1682-93. https://doi.org/10.1104/pp.010442.

78. Tsuji H, Aya K, Ueguchi-Tanaka M, Shimada Y, Nakazono M, Watanabe R, et al. GAMYB controls different sets of genes and is differentially regulated by microRNA in aleurone cells and anthers. Plant J. 2006;47(3):427-44. https://doi.org/10.1111/j.1365-313X.2006.02795.X.

79. Radley ME. Effect of abscisic acid and gibberellic acid on grain set in wheat. Ann Appl Biol. 1980;95(3):409-14. https://doi.org/10.1111/j.1744-7348.1980. tb04761.x.

80. Colombo N, Favret EA. The effect of gibberellic acid on male fertility in bread wheat. Euphytica. 1996;91(3):297-303. https://doi.org/10.1007/ BF00033091.

81. Millar AA, Gubler F. The Arabidopsis GAMYB-like genes, MYB33 and MYB65, are microRNA-regulated genes that redundantly facilitate anther development. Plant Cell. 2005;17(3):705-21. https://doi.org/10.1105/tpc.104. 027920

82. Csukasi F, Donaire L, Casañal A, Martínez-Priego L, Botella MA, MedinaEscobar N. Two strawberry miR159 family members display developmentalspecific expression patterns in the fruit receptacle and cooperatively regulate Fa-GAMYB. New Phytol. 2012;195(1):47-57. https://doi.org/10.1111/ j.1469-8137.2012.04134.x

83. Poznan Plant Breeding Station. Polish seed and breeding company. https:// phr.pl/en/. Accessed 16 May 2021.

84. BioProject PRJNA285604. Sequencing and analysis of Lupinus luteus transcriptome. https://www.ncbi.nlm.nih.gov/bioproject/?term=PRJNA2 85604. Accessed 28 Oct 2020

85. Schmittgen TD, Livak KJ. Analyzing real-time PCR data by the comparative C(T) method. Nat Protoc. 2008;3(6):1101-8. https://doi.org/10.1038/nprot.2 008.73 .

86. FastPCR - an integrated tool for PCR primers design. http://primerdigital. com/fastpcr.html. Accessed 28 Oct 2020.

87. The Universal Probe Library Assay Design Center. https://lifescience.roche com/en_pl/brands/universal-probe-library.html\#assay-design-center. Accessed 28 Oct 2020

88. Basic Local Alignment Search Tool. https://blast.ncbi.nlm.nih.gov/Blast.cgi. Accessed 28 Oct 2020

89. The bioinformatics resource portal of the Swiss Institute of Bioinformatics. http://www.expasy.org/. Accessed 28 Oct 2020.

90. Translate tool. https://web.expasy.org/translate/. Accessed 28 Oct 2020.

91. ProtParam tool. https://web.expasy.org/protparam/. Accessed 28 Oct 2020.

92. Phylogenetic analysis pipeline by ETE3. https://www.genome.jp/tools/ete/. Accessed 28 Oct 2020.

93. Multiple Sequence Alignment by CLUSTALW. https://www.genome.jp/toolsbin/clustalw. Accessed 28 Oct 2020

94. NCBI's Conserved Domain Database (CDD). (http://www.ncbi.nlm.nih.gov/ Structure/cdd/wrpsb.cgi). Accessed 28 Oct 2020.

95. TMHMM Server for Prediction of transmembrane helices in proteins. http:// www.cbs.dtu.dk/services/TMHMM/. Accessed 28 Oct 2020.

96. Phyre $^{2}$ - Protein Homology/analogy Recognition Engine. http://www.sbg. bio.ic.ac.uk/phyre2/html/page.cgi?id=index. Accessed 28 Oct 2020.

97. DiAlign: Local multiple alignment. http://www.genomatix.de/cgi-bin/dialign/ dialign.pl. Accessed 28 Oct 2020

98. Robetta - protein structure prediction service. http://new.robetta.org/. Accessed 28 Oct 2020

99. UCSF ChimeraX - visualization program from the Resource for Biocomputing, Visualization, and Informatics (RBVI). https://www.cgl.ucsf. edu/chimerax/. Accessed 28 Oct 2020.

100. Web Servers for RNA Secondary Structure Prediction. http://rna.urmc. rochester.edu/RNAstructureWeb. Accessed 28 Oct 2020.

\section{Publisher's Note}

Springer Nature remains neutral with regard to jurisdictional claims in published maps and institutional affiliations. 